\title{
Mixing lipids to manipulate the ionization status of lipid nanoparticles for specific tissue targeting
}

This article was published in the following Dove Press journal:

International Journal of Nanomedicine

\author{
Nour Shobaki* \\ Yusuke Sato* \\ Hideyoshi Harashima \\ Laboratory for Molecular Design \\ of Pharmaceutics, Faculty of \\ Pharmaceutical Sciences, Hokkaido \\ University, Sapporo, Hokkaido, Japan \\ *These authors contributed equally \\ to this work
}

Introduction: The development of targeted drug delivery systems is a rapidly growing area in the field of nanomedicine.

Methods: We report herein on optimizing the targeting efficiency of a lipid nanoparticle (LNP) by manipulating the acid dissociation constant $(p K a)$ value of its membrane, which reflects its ionization status. Instead of changing the chemical structure of the lipids to achieve this, we used a mixture of two types of $\mathrm{pH}$-sensitive cationic lipids that show different $p K a$ values in a single LNP. We mixed various ratios of YSK05 and YSK12-C4 lipids, which have $p K a$ values of 6.50 and 8.00, respectively, in one formulation (referred to as YSK05/12-LNP).

Results: The $p K a$ of the YSK05/12-LNP was dependent not only on the molar ratio of each lipid but also on the individual contribution of each lipid to the final $p K a$ (the YSK12-C4 lipid showed a higher contribution). Furthermore, we succeeded in targeting and delivering short interfering RNA to liver sinusoidal endothelial cells using one of the YSK05/12-LNPs which showed an optimum $p K a$ value of 7.15 and an appropriate ionization status ( $\sim 36 \%$ cationic charge) to permit the particles to be taken up by liver sinusoidal endothelial cells.

Conclusion: This strategy has the potential for preparing custom LNPs with endless varieties of structures and final $\mathrm{pKa}$ values, and would have potential applications in drug delivery and ionic-based tissue targeting.

Keywords: acid dissociation constant, liver sinusoidal endothelial cells, physical targeting, short interfering RNA

\section{Introduction}

Targeted delivery systems are promising tools for the specific delivery of therapeutic cargos into tissues of interest, with the objective of improving therapeutic outcomes while minimizing adverse effects on nontargeted tissues. Examples of targeting strategies include 1) passive targeting, in which delivery systems accumulate at the target site through the blood circulation; ${ }^{1,2}$ 2) active targeting, in which delivery systems are directed to specific sites through targeting moieties attached to their surface (eg, ligands or antibodies); $;^{3,4}$ and 3) physical targeting, in which delivery systems are targeted at certain environments with special physical characteristics such as $\mathrm{pH},{ }^{5}$ temperature, ${ }^{6}$ or ionic property. ${ }^{7,8}$ The objective of the present study was to investigate the physical targeting of a delivery system, based on the ionic charge and electrostatic interactions of the particle with cellular membranes.

Gene therapy using short interfering RNA (siRNA), a short sequence of nucleotides that can silence genes of interest, ${ }^{9,10}$ can be applied to the treatment of a wide variety of diseases related to genetic disturbances, such as cancer. However, the physical properties of siRNAs hinder their entry into cells: high molecular weight $(\sim 13,000 \mathrm{Da})$, hydrophilic nature, and highly anionic charge. Moreover, siRNAs are not stable in an in vivo environment. Therefore, an effective and safe siRNA drug delivery system is required.
Correspondence: Hideyoshi Harashima Laboratory for Molecular Design of Pharmaceutics, Faculty of Pharmaceutical Sciences, Hokkaido University, Kita-12, Nishi-6, Kita-ku, Sapporo, Hokkaido 060-08I2, Japan

$\mathrm{Tel}+8$ I I 7063919

Fax +8I II 7063734

Email harasima@pharm.hokudai.ac.jp 
Lipid nanoparticles (LNPs) have proved to be efficient systems for delivering siRNA into body tissues. ${ }^{11-15} \mathrm{We}$ assumed that by modifying the acid dissociation constant $(p K a)$ value of the LNP membrane, which reflects its ionization status, it might electrostatically interact with specific tissue membranes. Instead of changing the chemical structure of the lipids to manipulate the $p K a$ value of the LNP membrane, a method that is time-consuming and labor-intensive and results in limited $p K a$ adjustments, we mixed lipids having different $p K a$ values at different molar ratios in the LNP formulation, a method which minimized time and effort and allowed us to precisely adjust the final $p K a$ for achieving ionic-based tissue targeting.

Liver sinusoidal endothelial cells (LSECs) are an example of a tissue that would be a suitable model for our study and could be targeted based on the ionic charge of their surface. LSECs, which are the physical barrier that separate liver tissues from the blood flow, ${ }^{16,17}$ have significant roles under normal conditions, including providing a selective and permeable barrier, facilitating the transport of metabolites and waste clearance, ${ }^{18-20}$ regulating hepatic blood flow, maintaining a low portal pressure, ${ }^{21}$ and keeping hepatic stellate cells in their inactivated state to prevent fibrosis. ${ }^{22}$ However, the absence of any special characteristics and the phenotype of LSECs, due to capillarization (loss of fenestration and development of basement membrane), ${ }^{23}$ angiogenesis, ${ }^{24}$ or altered gene expression, ${ }^{25-27}$ results in the initiation and progression of various liver diseases, such as inflammation, fibrosis, cirrhosis, portal hypertension, and cancer metastasis..$^{24,27-31}$ Therefore, LSECs are possible drug targets for preventing or treating related liver disorders, especially those associated with genetic disturbances. Based on our previous observations, LSECs take up LNPs with higher $p K a$ values, in the $p K a$ range tested (5.70-7.25). ${ }^{32}$ However, higher $p K a$ values were not examined and the optimum $p K a$ value for targeting LSECs is not currently known.

We previously developed an LNP composed of different types of pH-sensitive cationic lipids, namely YSK lipids (referred to as YSK-LNP), to deliver siRNA in vitro and in vivo, particularly in hepatocytes. ${ }^{33,34}$ In our previous study, slightly cationic LNP formulations that were composed of lipids with high $p K a$ values, such as YSK13-C4 ( $p K a$ 6.80) and YSK15-C4 ( $p K a$ 7.10), were found to be highly localized in LSECs as opposed to hepatocytes. ${ }^{32}$ Nevertheless, they showed a weak gene silencing activity in LSECs, possibly due to their inactivation by endothelial lipase (EL). ${ }^{32}$ This prompted us to investigate the feasibility of targeting LSECs using lipase-resistant LNPs in which the membrane had optimized $p K a$ values.
As mentioned above, instead of synthesizing new lipids with new chemical structures and $p K a$ values, we prepared mixtures of lipids with different $p K a$ values in one LNP formulation at various molar ratios to manipulate the final $p K a$ of the membrane for LSECs targeting. The lipids used were YSK05 and YSK12-C4 ( $p K a 6.50$ and 8.00, respectively). Both lipids are lipase-resistant and have a strong endosomal escape and gene silencing activity. ${ }^{33,35,36}$ Mixing these lipids into one LNP formulation (referred to as a YSK05/12-LNP) enabled us to prepare a wide variety of formulations in which the membranes had different final $p K a$ values that were not merely dependent on the quantity of each lipid in the mixture, but also on the individual contribution of each lipid to the final $p K a$ value ( $35 \%$ vs $65 \%$ by YSK05 and YSK12-C4, respectively). As proof of our concept, we successfully targeted and delivered siRNA to LSECs using one of the YSK05/12-LNP formulations which had an optimum $p K a$ value ( 7.15$)$ and ionization property ( $\sim 36 \%$ cationic charge) for use in uptake by LSECs.

\section{Material and methods Materials and reagents}

YSK05 (1-methyl-4,4-bis(((9Z,12Z)-octadeca-9,12-dien1-yl)oxy)piperidine) and YSK12-C4 (6Z,9Z,28Z,31Z)-19(4-(dimethyl-amino)butyl)heptatriaconta-6,9,28,31 tetraen19-ol) lipids were synthesized in our laboratory as described previously. ${ }^{33,36}$ Cholesterol (Chol) was purchased from Sigma-Aldrich (St Louis, MO, USA). 1,2-Dimirystoyl-sn3-glycero methoxypolyethyleneglycol 2000 ether $\left({ }_{\mathrm{m}} \mathrm{PEG}_{2 \mathrm{k}}{ }^{-}\right.$ DMG) was purchased from the NOF Corporation (Tokyo, Japan). RiboGreen, DiI, and DiD were purchased from Molecular Probes (Eugene, OR, USA). The TRIzol reagent was purchased from Invitrogen (Carlsbad, CA, USA). The 6-( $p$-Toluidino)-2-naphthalenesulfonic acid (TNS) was purchased from Wako Pure Chemical Industries Ltd (Osaka, Japan). The sequences for the sense and antisense strands of siRNAs used in this study are listed in Table S1. All the siRNAs used in this study were obtained from Hokkaido System Science (Sapporo, Japan). The sequences for the forward and reverse primers used in this study are listed in Table S2. All primers were obtained from SIGMA Genosys Japan (Ishikari, Japan).

\section{Animals}

ICR ( 9,4 weeks, 16-22 g) mice were obtained from Japan SLC (Shizuoka, Japan). All animal experimental protocols were reviewed and approved by the Hokkaido University Animal Care Committee in accordance with the guidelines for the care and use of laboratory animals. 


\section{Preparation of LNPs}

The LNPs were prepared by a tertiary butanol $(t-\mathrm{BuOH})$ dilution procedure, as previously reported..$^{33,37,38}$ The lipids, which include $1050 \mathrm{nmol}$ of YSK lipids (YSK05 or YSK12-C4 or a mixture of both), $450 \mathrm{nmol}$ of cholesterol, and $30 \mathrm{nmol}$ of ${ }_{\mathrm{m}} \mathrm{PEG}_{2 \mathrm{k}}-\mathrm{DMG}$ - which represent a molar ratio of 70:30:2, respectively - were first dissolved in $400 \mu \mathrm{L}$ of $90 \%(\mathrm{v} / \mathrm{v})$ $t$ - $\mathrm{BuOH}$. When a fluorescent material (DiI or DiD) was incorporated into the LNPs, it was added at a concentration of $0.5-1 \mathrm{~mol} \%$ or $0.15 \mathrm{~mol} \%$ (of the total lipid) to the lipid solution. A $200 \mu \mathrm{L}$ portion of an aqueous solution containing $40 \mu \mathrm{g}$ siRNA was then added gradually to the lipid solution under vigorous mixing, producing an siRNA/lipid ratio of $0.042(\mathrm{wt} / \mathrm{wt})$. The siRNA-lipid solution was then gradually added to $2 \mathrm{~mL}$ of $20 \mathrm{mM}$ citrate buffer (pH 4.0) under vigorous mixing to facilitate the precipitation and formation of the LNPs. This yields a final $t$ - $\mathrm{BuOH}$ concentration of $60 \%(\mathrm{v} / \mathrm{v})$. Finally, ultrafiltration using Amicon ultracentrifugal tubes (Merck Millipore Ltd, Darmstadt, Germany) was performed to remove the $t \mathrm{BuOH}$, replace the external buffer with PBS (-) (pH 7.40), and concentrate the LNPs. Centrifugation was performed at $1,000 \times g$ for 11-18 minutes at RT.

\section{Characterization of LNPs}

The average diameter and $\zeta$-potential of LNPs were measured by dynamic light scattering (DLS) using Zetasizer Nano ZS ZEN3600 instrument (Malvern Instruments, Worcestershire, UK). The siRNA encapsulation efficiency, concentration, and recovery ratio were measured using the RiboGreen assay as previously described ${ }^{33}$ by which LNPs were diluted in $10 \mathrm{mM}$ HEPES buffer at $\mathrm{pH} 7.40$ containing $20 \mu \mathrm{g} / \mathrm{mL}$ dextran sulfate and RiboGreen in the presence or absence of $0.1 \mathrm{w} / \mathrm{v} \%$ Triton X-100. Fluorescence was measured using an Enspire 2300 multilabel reader (Perkin Elmer, Waltham, MA, USA) setup with $\lambda_{\mathrm{ex}}=500 \mathrm{~nm}$, $\lambda_{\mathrm{em}}=525 \mathrm{~nm}$. The siRNA concentration was calculated from the siRNA standard curve. The siRNA encapsulation efficiency was calculated by comparing siRNA concentration in the presence and absence of Triton X-100. The morphology of the LNPs was observed by transmission electron microscopy (TEM), in which a drop of an aqueous solution containing LNPs was adsorbed to carbon-coated copper grids (400 mesh) and the samples were stained with a $2 \%$ phosphotungstic acid solution ( $\mathrm{pH} 7.0$ ) for 20 seconds. The sample was observed by TEM (JEM-1400Plus, JEOL Ltd., Tokyo, Japan) at an acceleration voltage of $100 \mathrm{kV}$. Digital images $(3,296 \times 2,472$ pixels) were taken with a CCD camera (EM-14830RUBY2, JEOL Ltd.).

\section{Measurement of the $p K a$ value of the LNP membrane}

The $p K a$ value of the LNP membrane was measured by a TNS assay, as described previously. ${ }^{32,33}$ First, $0.5 \mathrm{mM}$ of LNP's lipid and $0.6 \mathrm{mM}$ of TNS (a negatively charged fluorescent dye) were mixed in $200 \mu \mathrm{L}$ of the following buffers (each buffer contained $150 \mathrm{mM} \mathrm{NaCl}$ ) $20 \mathrm{mM}$ citrate buffer ( $\mathrm{pH}$ 3.50-5.50), $20 \mathrm{mM}$ sodium phosphate buffer ( $\mathrm{pH} 6.00-8.00$ ), or $20 \mathrm{mM}$ Tris-HCl buffer (pH 8.00-9.00) in a 96-well plate, to yield a final concentration of 30 and $6 \mu \mathrm{M}$ of LNP's lipid and TNS in each well, respectively. Fluorescence was then measured using an Enspire 2300 multilabel reader (Perkin Elmer) setup with $\lambda_{\text {ex }}=321 \mathrm{~nm}, \lambda_{\text {em }}=447 \mathrm{~nm}$ at $37^{\circ} \mathrm{C}$. The $p K a$ was determined to be the $\mathrm{pH}$ value with $50 \%$ of maximal fluorescent intensity.

\section{Observation and quantification of biodistribution}

Mice, regardless of their body weight to minimize variation, were injected with $200 \mu \mathrm{L}$ of LNPs encapsulating $10 \mu \mathrm{g}$ siRNA (assuming a mouse body weight of $20 \mathrm{~g}$, the injected dose would be $0.5 \mathrm{mg}$ of siRNA/ $\mathrm{kg}$ ). In the LNPs, either the lipid was labeled with DiD or the siRNA was labeled with AF647. At 30 minutes after the injection, the mice were sacrificed and their body organs (liver, lung, kidneys, and spleen) were collected and the fluorescence of either the lipids or siRNA was observed using FluorVivo 300 small animal fluorescence imaging (INDEC BioSystems, Santa Clara, CA, USA). For quantifying the fluorescence intensity in each organ, the ImageJ software was used as follows: after selecting the region of interest (ROI) in each image, its corrected fluorescence was measured using the following formula:

\section{Corrected fluorescence \\ $=$ Integrated density $-($ Area of ROI \\ $\times$ Mean gray value of background reading)}

The corrected fluorescence of the treated samples was then normalized to the corrected fluorescence of PBS (-)-treated samples and then divided by the area of the ROI.

\section{Observation of intrahepatic distribution}

Mice were intravenously injected with DiI-labeled LNP encapsulating a mixture of siRNA (siGL4:siCy5-green fluorescent protein [GFP] 1:1 ratio) at a dose of $0.5 \mathrm{mg} / \mathrm{kg}$ of both siRNAs. After 1 hour, the mice were sacrificed, and their liver tissues $\left(0.5-1 \mathrm{~cm}^{2}\right)$ were collected and immersed in staining solution (Dulbecco's phosphate-buffered saline [D-PBS (-)]) containing $20 \mu \mathrm{g} / \mathrm{mL}$ FITC-labeled Isolectin 
B4 (Vector Laboratories, Burlingame, CA, USA) for staining blood vessels and $1 \mu \mathrm{g} / \mathrm{mL}$ Hoechst33342 (Dojindo, Kumamoto, Japan) for staining nuclei. Intrahepatic distribution of the LNPs was observed using confocal laser scanning microscopy (CLSM; Nikon A1, Nikon, Tokyo, Japan).

\section{Evaluation of gene silencing}

Mice were intravenously injected with the LNPs with a mixture of siRNA (siCD31:siFVII 1:1 ratio) encapsulated at a dose of $0.1 \mathrm{mg} / \mathrm{kg}$ of each type of siRNA. After 24 hours, the mice were sacrificed, and their body tissues were collected to evaluate the silencing of CD31 in the endothelium of various organs at the mRNA level as described in this section, and blood was collected to evaluate the silencing of plasma coagulation factor VII (FVII) at the protein level, as described below. To evaluate the silencing of CD31, $30 \mathrm{mg}$ samples of liver, lung, and spleen tissues were each homogenized using a Precellys 24 (Bertin technologies, Aix-en-Provence, France) in $500 \mu \mathrm{L}$ of TRIzol reagent, and the RNA was then extracted according to the manufacturer's protocol. The RNA was then converted to complementary DNA (cDNA) using a high-capacity RNA-to-cDNA kit (Applied Biosystems, Foster city, CA, USA) according to the manufacturer's protocol. Finally, a quantitative real-time polymerase chain reaction (qRT-PCR) analysis was performed on cDNA to evaluate gene expression using Fast SYBR Green Master Mix (Applied Biosystems) and Lightcycler 480 system II (Roche, Basel, Switzerland).

\section{Measurement of plasma coagulation factor VII activity}

At 24 hours after the mice had been injected with the LNPs encapsulating a mixture of siRNA (siCD31:siFVII 1:1 ratio) at a dose of $0.1 \mathrm{mg} / \mathrm{kg}$ of each type of siRNA as described in the previous section, blood was collected from their veins in the presence of heparin. The blood was then centrifuged at these conditions: $800 \times g, 5$ minutes, $4^{\circ} \mathrm{C}$. Plasma, the clear supernatant of the centrifuged blood, was removed and FVII protein expression was evaluated using a Biophen FVII kit (Hyphen Biomed, Neuville-Sur-Oise, France) according to the manufacturer's protocol.

\section{Evaluation of LNP-associated toxicity}

The LNP-associated toxicity was evaluated by measuring liver enzyme levels (aspartate transaminase [AST] and alanine transaminase [ALT]) using a GOT·GPT CII kit (Wako Pure Chemical Industries Ltd) according to the manufacturer's protocol. Furthermore, inflammatory cytokine gene expressions (interleukin-6 [IL-6] and tumor necrosis factor-alpha [TNF- $\alpha]$ ) were quantified by performing qRTPCR analysis on cDNA prepared from liver tissues using the same method described in Evaluation of gene silencing section in this article.

\section{Statistical analysis}

The results are expressed as the mean $\pm \mathrm{SD}$ of independent repeats. Statistical comparisons between groups were evaluated by nonrepeated measures ANOVA followed by SNK test.

\section{Results}

\section{Physical characteristics of LNPs}

Various LNPs (referred to as YSK05/12-LNPs) were prepared using the $t-\mathrm{BuOH}$ dilution procedure by mixing two different lipids YSK05 and YSK12-C4 ( $p K a 6.50$ and 8.00, respectively) as follows: YSK05/YSK12-C4/cholesterol/ ${ }_{\mathrm{m}} \mathrm{PEG}_{2 \mathrm{k}}-\mathrm{DMG}=(70-X): X: 30: 2 \mathrm{~mol} \%$ of total lipids $(X=0-70$; Figure 1). Typically, the LNPs have diameters in the range of 70-100 nm with a homogenous size distribution (polydispersity index $[\mathrm{PDI}]<0.2$ ), and the encapsulation of siRNA exceeds $90 \%$. The $\zeta$-potential at $\mathrm{pH} 7.40$ varied from neutral to cationic, depending on the type of YSK lipid used and the amount in the LNP. An example of measuring the LNP's particle size and $\zeta$-potential by DLS and observing its morphology by TEM is shown in Figure 2. Further characterization data for the LNPs with various lipid compositions are shown in Table S3.

\section{Manipulating the $p K a$ value of the LNP membrane through mixing lipids}

The $p K a$ values for the YSK05/12-LNP membranes were determined using a TNS assay. TNS is a compound that electrostatically interacts with the cationic lipid membrane, resulting in fluorescence,,$^{39,40}$ and the $p K a$ of the LNP was determined to be the $\mathrm{pH}$ value with 50\% maximal fluorescent intensity. This method was found to be a better alternative for $\zeta$-potential measurement to predict the real charge state of the LNP surface in the in vivo environment. As shown in Figure 3, mixing YSK05 and YSK12-C4 ( $p K a$ 6.50 and 8.00 , respectively) at different molar ratios resulted in LNPs in which the membrane had different final $p K a$ values, ranging between 6.50 and 8.00 .

\section{The pKa value of the LNP membrane was not merely dependent on the quantity of lipid used}

The relationship between lipid quantity or the molar ratio of each of the lipid components and the final $p K a$ 
A
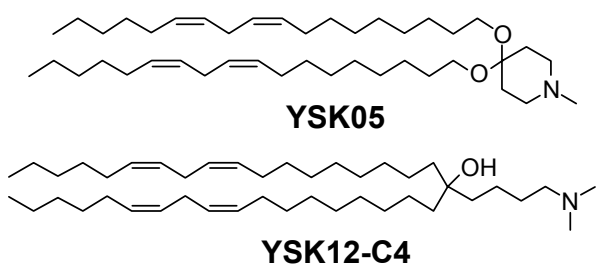

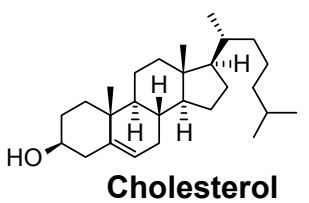

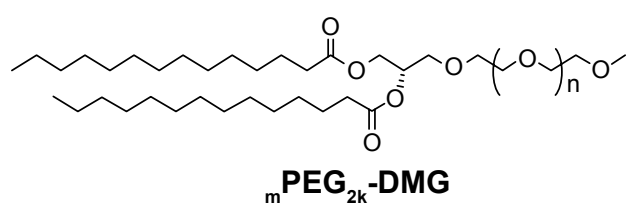

B

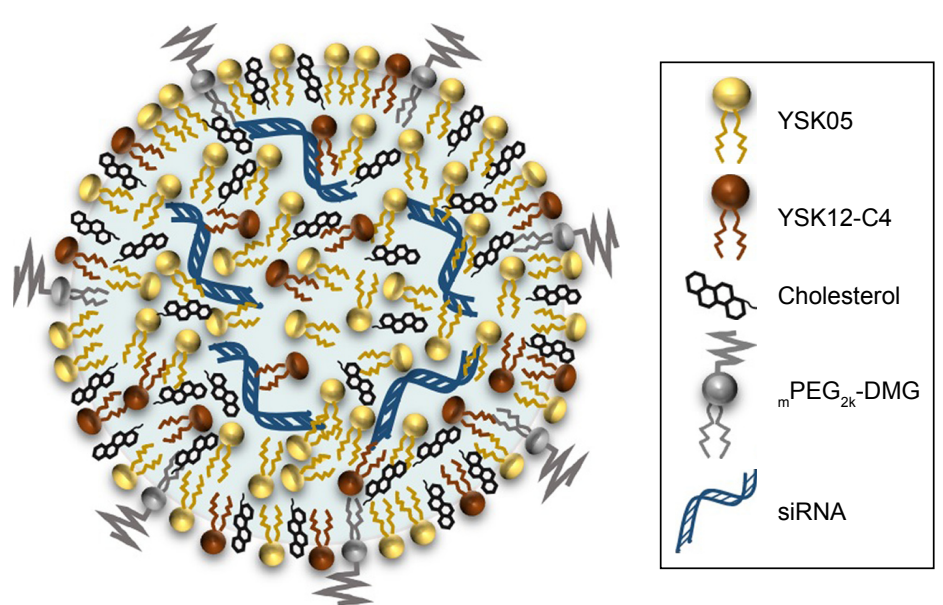

Figure I Composition and structure of the YSK05/I2-LNPs

Notes: (A) The chemical structure of the lipid components in the LNPs used in this study. (B) A schematic illustration of LNP structure. The YSK05/I2-LNP was prepared by means of $t$-BuOH dilution method by mixing the following different molar ratios of YSK05 and YSK I2-C4 lipids: YSK05/YSK I 2-C4/Cholesterol/ ${ }_{\mathrm{m}}$. mol\% of total lipids $(X=0-70)$.

Abbreviations: LNP, lipid nanoparticle; ${ }_{m} P E_{2 k}$-DMG, I,2-Dimirystoyl-sn-3-glycero methoxypolyethyleneglycol 2000 ether; siRNA, short interfering RNA; $t$-BuOH, tertiary butanol.
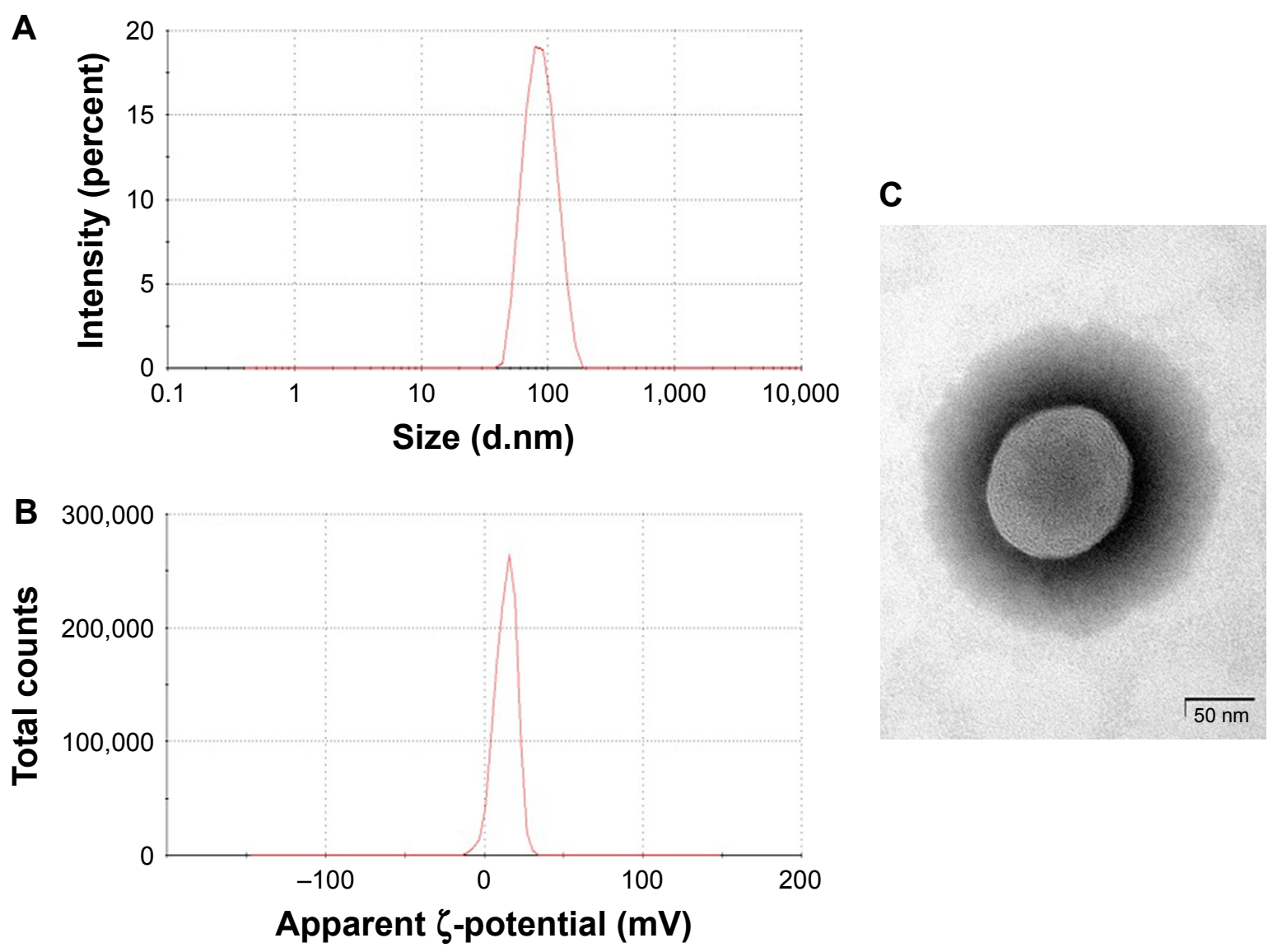

Figure 2 Physical characteristics of the YSK05/I2-LNP.

Notes: Particle size (A) and $\zeta$-potential distribution (B), as detected by DLS and the morphology of LNPs, as observed by TEM (C). The YSK05/I2-LNP used for these measurements was prepared by means of the $t-\mathrm{BuOH}$ dilution method. It has the following composition: $\mathrm{YSK} 05 / \mathrm{YSK} / 2-\mathrm{C} 4 / \mathrm{Cholesterol} / \mathrm{m} P \mathrm{P}_{2 \mathrm{k}}-\mathrm{DMG}=50: 20: 30: 2 \mathrm{~mol} \%$ of total lipids and the following characteristics: size, $82.75 \mathrm{~nm}$; number mean, $63.24 \mathrm{~nm}$; PDI, 0.079; $\zeta$-potential, I3.5 mV; EE of siRNA, $99.46 \%$. Scale bars: $50 \mathrm{~nm}$ (C).

Abbreviations: DLS, dynamic light scattering; EE, encapsulation efficiency; LNP, lipid nanoparticle; ${ }_{m} P G_{2 k}$-DMG, I,2-Dimirystoyl-sn-3-glycero methoxypolyethyleneglycol 2000 ether; PDI, polydispersity index; siRNA, short interfering RNA; $t-\mathrm{BuOH}$, tertiary butanol; TEM, transmission electron microscopy. 


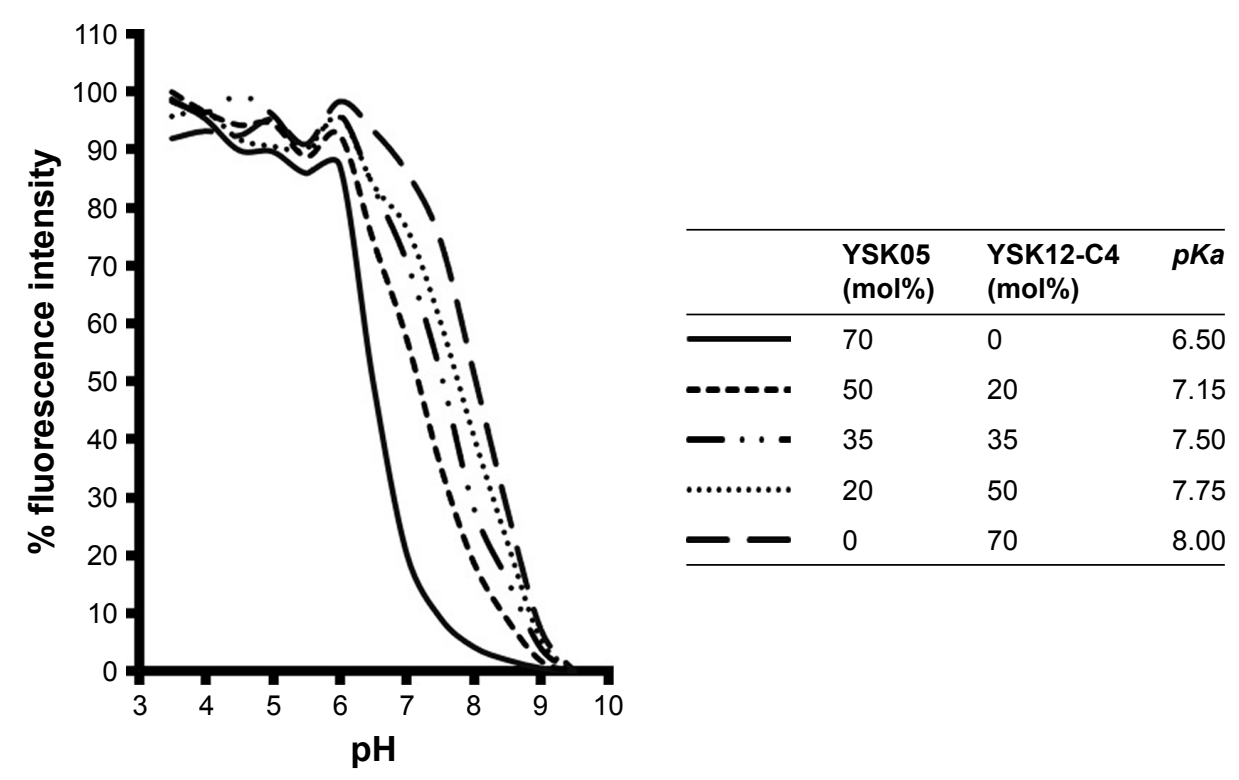

Figure 3 The YSK05/I2-LNP fluorescence intensity under different $\mathrm{pH}$ values measured by TNS assay.

Notes: The $p K a$ value of the LNP membranes was determined to be the $\mathrm{pH}$ value with $50 \%$ of maximal fluorescence intensity. Data represent the mean ( $n=3$ ).

Abbreviations: LNP, lipid nanoparticle; $p K a$, acid dissociation constant; TNS, 6-( $p$-toluidino)-2-naphthalenesulfonic acid.

value for the membrane was expected to be linear, as represented in Figure 4A, dotted line. In other words, the $p K a$ value or the LNP membrane was expected to be an average of the $p K a$ values of its lipid components, depending on their quantity, which can be calculated using Equation 1 considering two factors: the $p K a$ value of each lipid and its molar ratio in the LNP.

$$
p K a_{1+2}=\frac{\left(\left(p K a_{1} \times X\right)+\left(p K a_{2} \times(1-X)\right)\right)}{(X+(1-X))}
$$

where $p K a_{1}$ is the $p K a$ of first lipid (eg, YSK12-C4), $p K a_{2}$ is the $p K a$ of second lipid (eg, YSK05), $p K a_{1+2}$ is the $p K a$ of the LNP (eg, YSK05/12-LNP), $X$ is the molar ratio of one lipid (eg, YSK12-C4).
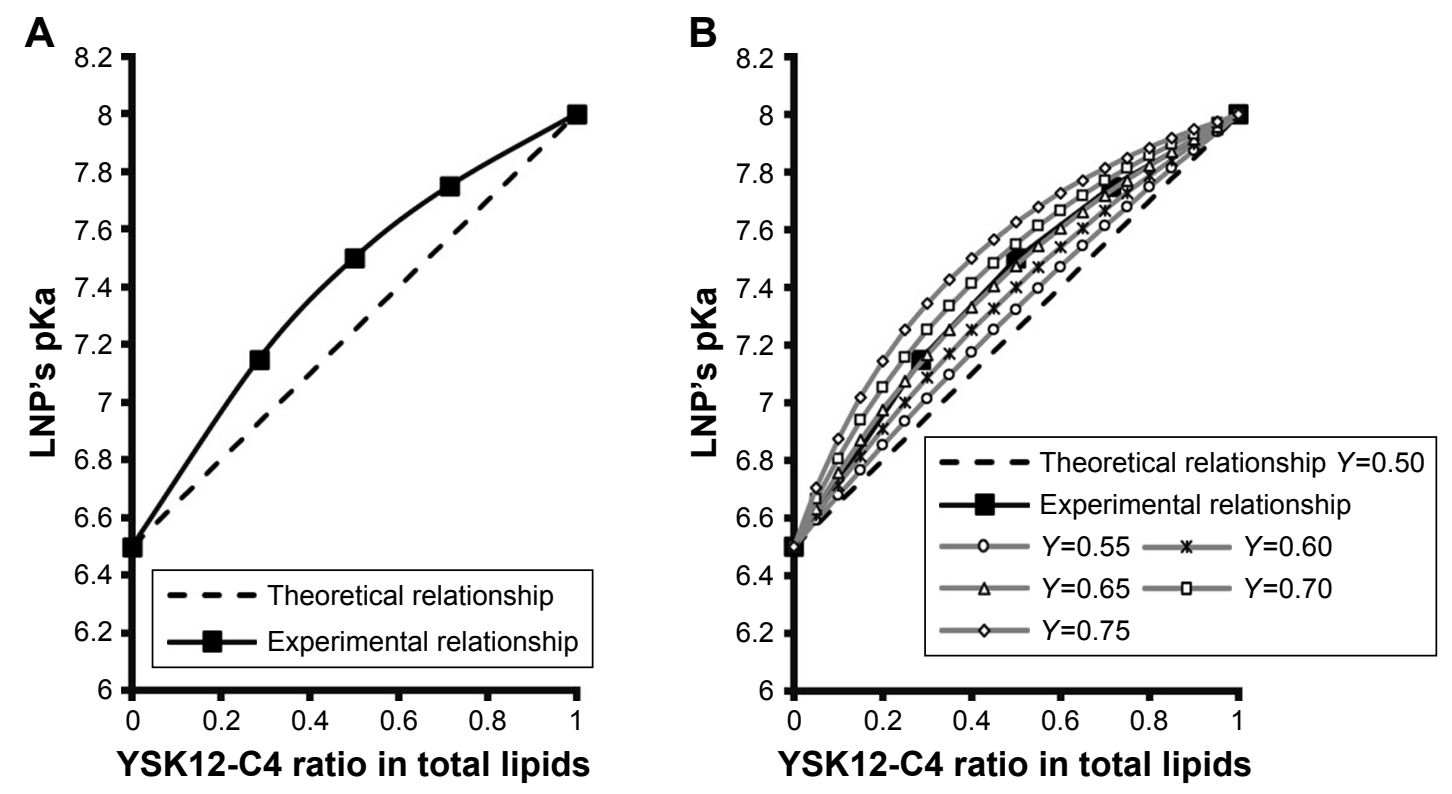

Figure 4 The relationship between lipid quantity and the pKa value of the LNP membrane.

Notes: (A) The relationship between the YSK I2-C4 ratio in total lipids (X/70) and the pKa value for the YSK05/I2-LNP membrane, theoretically simulated or experimentally measured using Equation I or TNS assay, respectively. (B) Determination of $Y$ value (contribution factor) of YSK I2-C4 lipid in the modified Equation 2.

Abbreviations: LNP, lipid nanoparticle; TNS, 6-( $p$-toluidino)-2-naphthalenesulfonic acid; $p K a$, acid dissociation constant. 
However, the experimental results indicated that this was not the case, in that, the relationship between the molar ratio of each of the LNP's lipid components and the final $p K a$ value for the membrane exhibited a nonlinear relationship, in which YSK12-C4 ( $p K a$ 8.00) had a higher contribution to the final $p K a$ value than YSK05 ( $p K a$ 6.50) (Figure 4A, line with squares). Therefore, the equation was modified to Equation 2 by adding a third factor, the $Y$ factor (contribution factor), which represents the contribution of each lipid in the $p K a$ value for the LNP membrane.

$$
p K a_{1+2}=\frac{\left(\left(p K a_{1} \times X \times Y\right)+\left(p K a_{2} \times(1-X) \times(1-Y)\right)\right)}{((X \times Y)+((1-X) \times(1-Y)))}
$$

where $p K a_{1}$ is the $p K a$ of first lipid (eg, YSK12-C4), $p K a_{2}$ is the $p K a$ of second lipid (eg, YSK05), $p K a_{1+2}$ is the $p K a$ of the LNP (eg, YSK05/12-LNP), $X$ is the molar ratio of one lipid (eg, YSK12-C4), and $Y$ is the contribution of one lipid (eg, YSK12-C4).

If the relationship between the molar ratio of each of the LNP's lipid components and its final membrane's $p K a$ value was linear, then the $Y$ value of each lipid should be equal to 0.50 , which represents a $50 \%$ contribution of each lipid to the final $p K a$ value for the LNP membrane. However, because the experimentally found relationship was nonlinear, the $Y$ value of both lipids is different. To determine the $Y$ value (eg, of the YSK12-C4 lipid) in the experimental nonlinear relationship, various values $>0.50$ but $<1.00$ were examined. These values were chosen to be $>0.50$ because the YSK12-C4 lipid had a higher contribution than YSK05 to the final $p K a$ value for the LNP, as observed in Figure 4A, the line with squares. The chosen $Y$ values were then tested in Equation 2 to produce simulated relationships as shown in Figure 4B.

The $Y$ value that was found to fit completely into the equation to produce a simulated relationship identical to the experimental relationship measured by the TNS assay was 0.65 (Figure 4B, gray line with open triangles). In other words, YSK12-C4 lipid ( $p K a 8.00$ ) contributes $65 \%$ of the $p K a$ value for the YSK05/12-LNP membrane, while the YSK05 lipid ( $p K a$ 6.50) contributes the remainder, ie, $35 \%$.

\section{Gene silencing activity in the liver is dependent on the $p K a$ value of its membrane}

Mice were intravenously injected with YSK05/12-LNPs in which the membranes had different $p K a$ values, as shown in Figure 3. Gene knockdown activity in hepatocytes was observed for LNPs that contained the YSK05 lipid only, with a $p K a$ value of 6.50. This type of LNP induced approximately an $80 \%$ gene silencing of the FVII protein (a hepatocyte marker that has been used by us and other researchers as a reliable indicator of gene silencing activity of LNPs in the liver) ${ }^{34,37,43}$ with a $0.1 \mathrm{mg} / \mathrm{kg}$ dose of siFVII (Figure $5 \mathrm{~A}$ ). On the other hand, in LSECs, although LNPs composed of the YSK05 lipid only or the YSK12-C4 lipid produced only a weak activity, mixing these lipids in one LNP formulation
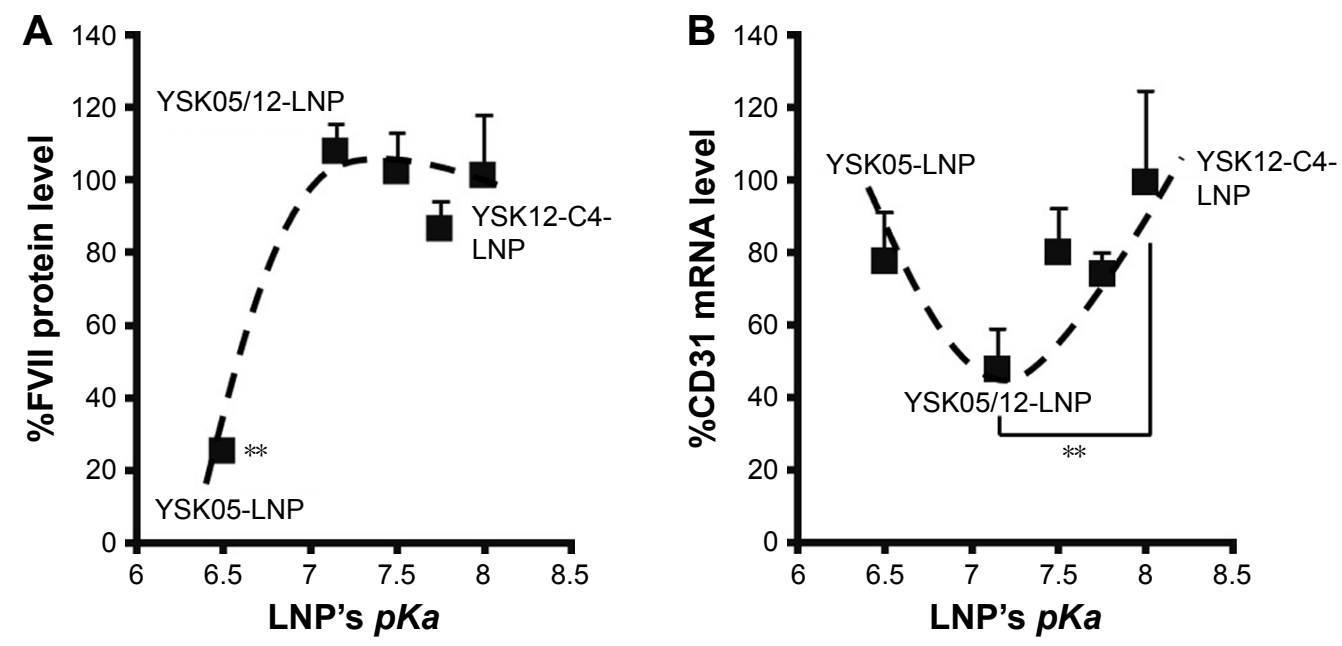

Figure $\mathbf{5}$ In vivo silencing activity of LNPs in hepatocytes and LSECs.

Notes: Mice were intravenously injected with LNPs encapsulating a mixture of siRNA (siCD3 I:siFVII I:I ratio) at a dose of 0.1 mg/kg of each type of siRNA. After 24 hours, levels of the FVII protein in plasma (A) and CD3I mRNA in liver tissues (B) were measured by means of an FVII assay and qRT-PCR, respectively. The reference gene in LSECs was TIE2. $* * P<0.0$ I, nonrepeated ANOVA followed by an SNK test; data represent the mean \pm SD $(n=3)$.

Abbreviations: FVII, coagulation factor VII; LNP, lipid nanoparticle; LSECs, liver sinusoidal endothelial cells; mRNA, messenger RNA; pKa, acid dissociation constant; qRT-PCR, quantitative real-time polymerase chain reaction; siRNA, short interfering RNA; TIE2, angiopoietin receptor. 

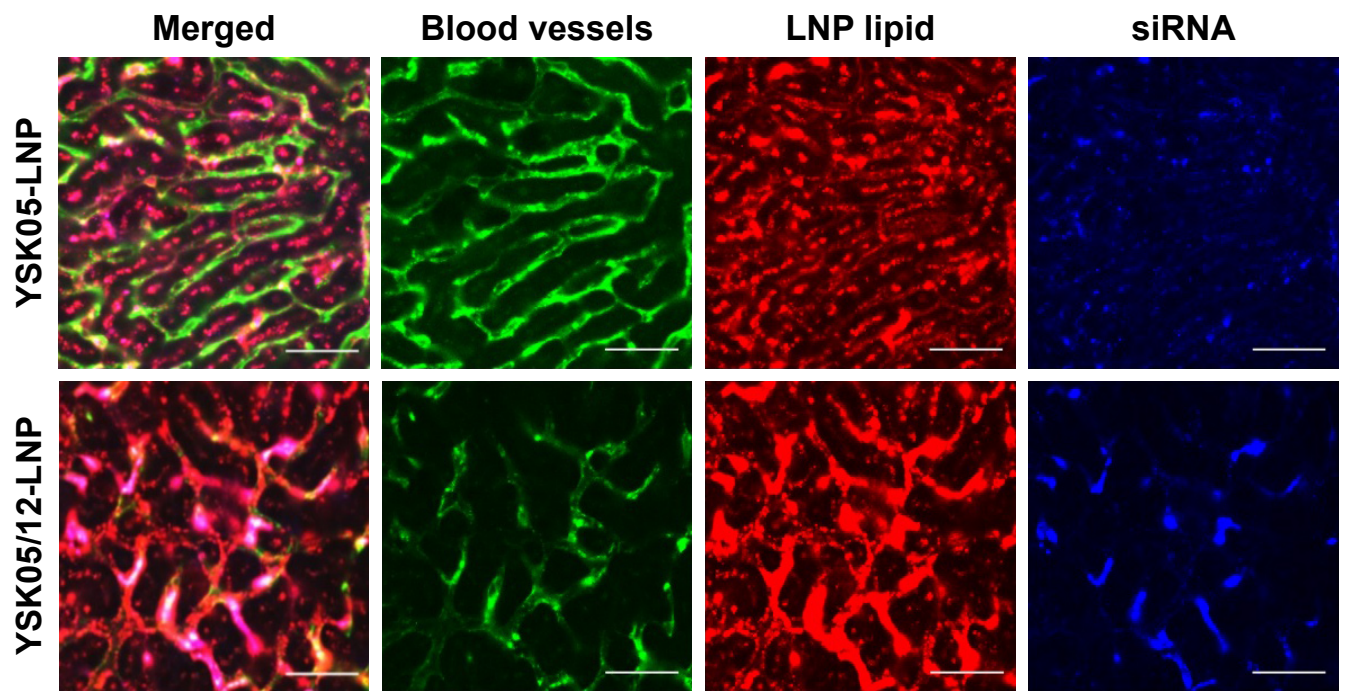

Figure 6 Intrahepatic distribution of YSK05-LNP (pKa 6.50) and YSK05/I2-LNP (pKa 7.I5).

Notes: Mice were intravenously injected with Dil-labeled LNPs encapsulating a mixture of siRNA (siGL4:siCy5-GFP I:I ratio) at a dose of 0.5 mg/kg of both siRNAs. After I hour, liver tissues were collected and the distribution of LNPs was observed by CLSM. Green color represents blood vessels/LSECs (FITC-Isolectin B4), red color represents LNP (Dil), and blue color represents siRNA (Cy5). Scale bars: $50 \mu \mathrm{m}$.

Abbreviations: CLSM, confocal laser scanning microscopy; FITC, fluorescein isothiocyanate; GFP, green fluorescent protein; LNP, lipid nanoparticle; LSECs, liver sinusoidal endothelial cells; siRNA, short interfering RNA.

optimized the $p K a$ value for the membrane and improved the LNP silencing activity to an approximately $60 \%$ knockdown of CD31 mRNA (an endothelial cell marker) with a $0.1 \mathrm{mg} / \mathrm{kg}$ dose of siCD31 (Figure 5B). The YSK05/12-LNP with the composition YSK05/YSK12-C4/cholesterol/ ${ }_{\mathrm{m}} \mathrm{PEG}_{2 \mathrm{k}}{ }^{-}$ $\mathrm{DMG}=50: 20: 30: 2 \mathrm{~mol} \%$ of total lipids resulted in the best mRNA silencing activity in LSECs, and the $p K a$ value for its membrane was 7.15 , thus confirming this to be optimal for specific uptake by LSECs. Furthermore, this optimized LNP had a minimal activity in other organ endothelia, such as the lung and spleen (Figure S1).

\section{Optimizing the pKa value of the LNP membrane improves its targeting efficiency}

The FluorVivo imaging (Figure S2) revealed that the YSK05-LNP ( $p K a$ 6.50) and YSK05/12-LNP ( $p K a 7.15$ and composition of YSK05/YSK12-C4/cholesterol/ ${ }_{\mathrm{m}} \mathrm{PEG}_{2 \mathrm{k}}{ }^{-}$ $\mathrm{DMG}=50: 20: 30: 2 \mathrm{~mol} \%$ of total lipids) have a similar biodistribution pattern and they are largely distributed to liver tissues. However, we decided to compare their intrahepatic distribution by observing liver tissues that had been injected with DiI-labeled LNP encapsulating a mixture of siRNA (siGL4:siCy5-GFP 1:1 ratio) at a dose of $0.5 \mathrm{mg} / \mathrm{kg}$ of both siRNAs using CLSM. Although the injected siRNA dose was higher than that used for obtaining gene silencing ( 0.5 vs $0.1 \mathrm{mg} / \mathrm{kg}$, respectively), it was selected to enable us to visualize hepatic distribution with high sensitivity and clarity.
We observed the LNP containing the YSK05 lipid only (YSK05-LNP) and a $p K a$ of 6.50 was extensively taken up by hepatocytes (Figure 6, upper). In the image, the YSK05LNP (represented in red color) is extensively distributed in hepatocytes, which are the most abundant cells in the liver and are defined in the image as the nonfluorescent areas that are infiltrated with LSECs which are shown in green color. On the other hand, the YSK05/12-LNP with a $p K a$ value of 7.15 was highly taken up by LSECs (Figure 6, lower). In the image, YSK05/12-LNP (represented in red) is distributed on LSECs (the green color of LSECs turned into red or orange because of LNP accumulation in this area). These results suggest that, despite the similar hepatic biodistribution of YSK05-LNP and YSK05/12-LNP, as shown in Figure S2, their intrahepatic distribution varied depending on their $p K a$ value.

It is noteworthy that no increase in the levels of liver enzymes (AST or ALT) was observed after intravenously injecting the mice with YSK05/12-LNP (Figure 7). Furthermore, inflammatory cytokine gene expression (for IL-6 and TNF- $\alpha$ ) was quantified in the liver tissues of the same group of mice by qRT-PCR; no increase in expression was found (data not shown). These data suggest the safety and lack of toxicity of the LNPs.

\section{Discussion}

The aim of this study was to improve the targeting efficiency of an LNP by optimizing the dissociation constant ( $p K a)$ 


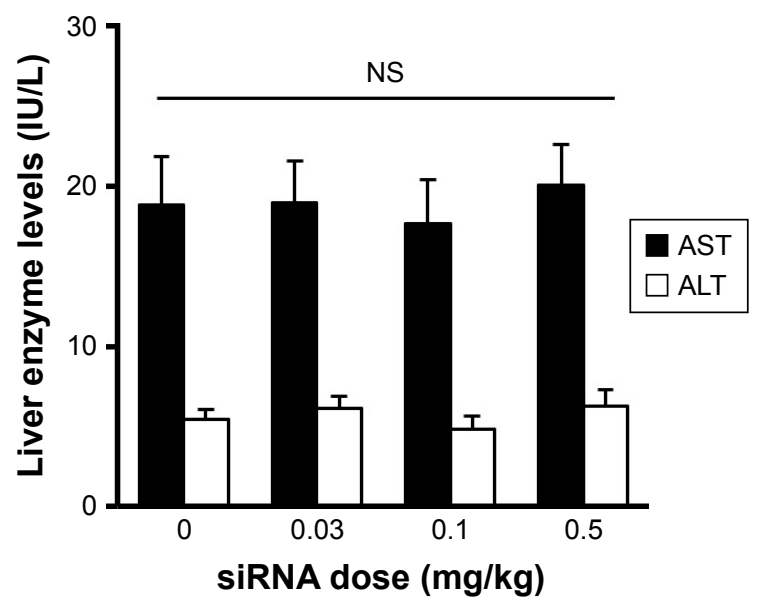

Figure 7 Liver enzyme levels after YSK05/I2-LNP injection.

Notes: Mice were intravenously injected with YSK05/I2-LNP. After 24 hours, plasma was collected, and liver enzymes were evaluated using a GOT GPT Cll kit. Nonrepeated ANOVA followed by an SNK test; data represent mean $\pm S D(n=3)$. Abbreviations: ALT, alanine transaminase (GPT); AST, aspartate transaminase (GOT); LNP, lipid nanoparticle; NS, not significant; siRNA, short interfering RNA.

value for its membrane, which reflects its ionization property or surface charge. This would permit electrostatic interactions with targeted tissues since both structures would have complementary charges. This could selectively enhance the uptake of LNPs by tissues of interest with minimal distribution to others, in a process that is called ionic-based physical targeting.

We previously reported on the development of our original LNPs, which can be prepared using several types of lipids that were synthesized in our laboratory, and are referred to as YSK lipids, to deliver siRNA to cells, both in vitro and in vivo. ${ }^{32-38}$ LNPs, especially those containing lipids, namely YSK05 and YSK13-C3, showed a high cellular uptake, strong endosomal escape, and efficient siRNA delivery and silencing activity, especially in hepatocytes. ${ }^{33,34} \mathrm{We}$ also found that the $p K a$ value of the LNP membrane influences its intrahepatic distribution. For example, LNPs that contain lipids with low $p K a$ values (eg, YSK05 and YSK13-C3 with $p K a$ values of 6.50 and $6.45 p K a$, respectively) are distributed in hepatocytes. Whereas, those containing lipids with higher $p K a$ values (eg, YSK13-C4 and YSK15-C4 with $p K a$ values of 6.80 and 7.10, respectively) are distributed in LSECs, ${ }^{32}$ which are border cells that are located between hepatocytes and the blood stream. ${ }^{16,17}$ However, due to the limited range of $p K a$ values (5.70-7.25) that were tested in that previous study ${ }^{32}$ higher $p K a$ values were not evaluated and the optimal $p K a$ value for an LNP for uptake by LSECs was not confirmed. Furthermore, despite the enhanced $p K a$-dependent distribution of YSK13-C4- and YSK15-C4-LNPs in LSECs, the silencing activity in those cells was relatively weak, probably due to their inactivation by EL which are distributed in LSECs and have phospholipase activity that cleaves the ester linkage present in those lipids. ${ }^{32,41}$ Inactivation by other lipases such as lipoprotein lipase or hepatic lipase can be excluded because they have triglyceride lipase activity.

We selected LSECs as a target tissue in this study for two reasons: first, the potential for LSEC targeting using LNPs containing a membrane with an optimized $p K a$ value ${ }^{32}$ would confirm the feasibility of our concept regarding ionic-based physical targeting. Second, the significance of LSECs in liver pathophysiology qualifies them as a potential drug target candidate for treating various liver disorders, ${ }^{18-31}$ especially those related to genetic disturbances that could be corrected by siRNA-based therapeutics and gene silencing. To improve the activity of YSK13-C4- and YSK15-C4-LNPs in LSECs, it was necessary to change the ester linker in those lipids to prevent their inactivation by EL. However, changing the linker type might hinder the ability of those lipids to induce efficient endosomal escape. It was reported elsewhere that the presence of an ester linker facilitates the lipid mixing mechanism with endosomal membranes, thus leading to an efficient endosomal escape of cargos even without the need to add helper fusogenic lipids. ${ }^{42,43}$ Another solution would be to preserve the ester linkages but inhibit EL activity. However, EL inhibitors such as GSK264220A might induce systemic side effects due to their interference with the physiological roles of EL in lipid metabolism. ${ }^{44}$ Therefore, it would be more efficient to develop an LNP formulation for which the $p K a$ value of the membrane would be optimal for targeting LSECs using lipase-resistant lipids.

There are two possible strategies for optimizing the $p K a$ of an LNP membrane for LSECs targeting. The first strategy is the synthesis of various lipids with different chemical structures and $p K a$ values for use in preparing LNPs with a wide range of membrane $p K a$ values and screen the uptake and activity of each in LSECs. However, this method is time- and effort-consuming, not cost-effective, and results in limited $p K a$ adjustments. Therefore, we adopted a more effective strategy, which involved mixing two lipids at different molar ratios with low and high $p K a$ values in several LNP formulations to permit the $p K a$ value of the final LNP membrane. These lipids are YSK05 (a pH-sensitive cationic lipid with a $p K a$ value of 6.50), and YSK12-C4 (a highly cationic lipid with a $p K a$ value of 8.00). Their chemical structures are shown in Figure 1. These lipids were chosen because they have a strong endosomal escape, strong activity (YSK05 in hepatocytes, in vivo, ${ }^{33}$ and YSK12-C4 in dendritic cells, in vitro $\left.{ }^{36}\right)$, and are resistant to the action of lipases. 
Several formulations (referred to as YSK05/12-LNPs) were prepared with the following composition: YSK05/ YSK12-C4/cholesterol/ $/ \mathrm{PEG}_{2 \mathrm{k}}-\mathrm{DMG}=(70-X): X: 30: 2 \mathrm{~mol} \%$ of total lipids ( $X=0-70$; Figure 1). As shown in Figure 2 and Table S3, the YSK05/12-LNPs, which were prepared using the $t-\mathrm{BuOH}$ procedure, typically have a diameter in the range of 70-100 $\mathrm{nm}$ with a homogenous size distribution $(\mathrm{PDI}<0.2)$, and the encapsulation of siRNA exceeds $90 \%$. The $\zeta$-potential distribution of the LNPs at $\mathrm{pH} 7.40$ was varied depending on the type of YSK lipid used and the amount in the LNP. Generally, the $\zeta$-potential value of the LNP shifts from neutral (when only the YSK05 lipid was used) to cationic (when only the YSK12-C4 lipid was used or mixed with YSK05 lipid). The $p K a$ values of the YSK05/12LNP membrane were measured by the fluorescent intensity of TNS, a compound that fluoresces only when it interacts with cationic lipid membranes. ${ }^{39,40}$ As we reported in a previous study, ${ }^{32}$ the apparent $p K a$ value of the LNP, which was determined to be the $\mathrm{pH}$ with $50 \%$ of the maximal fluorescence intensity of TNS, reflects the real charge state or ionization property of the LNP membrane in an in vivo environment, and is a better alternative to the $\zeta$-potential value which could be influenced by nonencapsulated negatively charged siRNA and/or PEG-DMG molecules that are attached to the LNP surface in the buffer while the $\zeta$-potential is being measured but are subsequently detached in the blood circulation.

It was previously reported that the $\mathrm{pH}$-sensitivity of the delivery system is a key parameter for its efficiency in delivering siRNA. ${ }^{33,45}$ An efficient delivery system should be able to sense small $\mathrm{pH}$ changes in the environment, such as the neutral $\mathrm{pH}$ in the blood and the acidic $\mathrm{pH}$ in cellular endosomes, and to switch its charge to a cationic charge in the endosome for efficient endosomal escape. As shown in Figure 3, mixing the lipids in the LNP formulation had no effect on its $\mathrm{pH}$-sensitivity, but rather it influenced the $p K a$ of the membrane and permitted the preparation of LNP formulations with $p K a$ values between 6.50 and 8.00 at various lipid molar ratios. As a result, lipid mixing was found to be an effective strategy for manipulating the final $p K a$ value of the LNP.

Before mixing the lipids, we expected to see a linear relationship between each lipid molar ratio and the final LNP's $p K a$ value (Figure 4A, dotted line), in which both lipids would contribute equally to the final $p K a$, and the final $p K a$ can be calculated using a simple mathematical equation that considers two factors: the $p K a$ value of each lipid and its molar ratio in the formulation (Equation 1). However, the experimental relationship, as measured by a
TNS assay was unexpectedly nonlinear (Figure 4B, line with squares), in which YSK12-C4 lipid ( $p K a$ 8.00) had a higher impact and contribution to the final $p K a$ for the LNP than YSK05 ( $p K a$ 6.50). Therefore, the equation was modified by adding a third factor called the $Y$ factor or contribution factor (Equation 2). In the linear simulated relationship, the $Y$ factor equals 0.50 , meaning that each lipid contributes equally or by $50 \%$ to the final $p K a$ value for the LNP. To determine the $Y$ value in the experimental relationship and specifically for the YSK12-C4 lipid which had a higher contribution in the final $p K a$, we investigated a series of values $>0.50$ and $<1.00(0.50<Y<1.00)$, which represents the percentage of the lipid contribution $(50 \%-100 \%)$ in the final $p K a$ value. The $Y$ value that was found to fit completely into the equation to produce a relationship identical to the experimental relationship was 0.65 (Figure 4B, gray line with open triangles). This indicates that YSK12-C4 contributes to the final $p K a$ by $65 \%$, while the contribution by YSK05 is $35 \%$. The reasons for this difference remain to be investigated in a future study. It is noteworthy that we previously encountered this nonlinear relationship when we used two different lipids, namely, YSK13-C2 and YSK13-C4 ( $p K a 5.70$ and 6.80, respectively) in one LNP formulation. The YSK13-C4 lipid contributed to a higher extent, by $73 \%$, to the final $p K a$ value of the LNP (the $Y$ value measured using Equation 2 was 0.73 ), while the YSK13-C2 lipid contributed the rest, ie, 27\%, to the final LNP's $p K a$ ([n=2], data not shown). We cannot, however, conclude that Equation 1 or 2 can be applied universally. More studies will need to be carried out using different kinds of lipids with different $p K a$ values to confirm that this is a universal equation that predicts the final $p K a$ value of the LNP resulting from lipid mixing.

After preparing YSK05/12-LNPs in which the membranes have different $p K a$ values, the in vivo knockdown activity of these LNPs was evaluated. Basically, the intrahepatic activity of YSK05/12-LNPs was compared with that of the original YSK05- or YSK12-C4-LNPs (the $p K a$ values for all these preparations are shown in Figure 3). The formulation that induced the strongest knockdown activity in hepatocytes was YSK05-LNP ( $p K a$ 6.50), while the activities for the YSK05/12-LNPs and YSK12-C4-LNP, which have higher $p K a$ values, were considerably lower (Figure $5 \mathrm{~A}$ ). A possible reason behind the hepatocyte uptake of YSK05-LNP might be related to the fact that hepatocytes preferentially take up neutral particles with lower $p K a$ values through the apolipoprotein E-low density lipoprotein receptor pathway, and this coincides with previous reports. ${ }^{32,43}$ On the other 
hand, in LSECs, despite the weak activity of both YSK05and YSK12-C4-LNPs, using mixtures in YSK05/12-LNP dramatically improved their activity in LSECs as the result of optimizing the $p K a$ value for the LNP membrane to 7.15 (Figure 5B). A possible reason behind this is the preference of endothelial cells to take up slightly cationic particles which electrostatically interact with the slightly anionic charge of the endothelial membrane.

The YSK05/12-LNP formulation which is composed of YSK05/YSK12-C4/cholesterol/ ${ }_{\mathrm{m}} \mathrm{PEG}_{2 \mathrm{k}}$-DMG (50:20:30:2 $\mathrm{mol} \%$ ) and a $\mathrm{kKa}$ value of 7.15 was chosen for use in experiments to target LSECs and compared with YSK05-LNP. Although YSK05-LNP and YSK05/12-LNP have a similar biodistribution, as shown in Figure S2, in which the lipids and siRNA of both types of LNPs were visualized by FluorVivo imaging and were found to be largely distributed to liver tissues, the intrahepatic distribution of those LNPs varied and was determined by their $p K a$ value. The intrahepatic distribution was shifted substantially from hepatocytes (with YSK05-LNP [ $p K a$ 6.50; Figure 6, upper]) into LSECs (with YSK05/12-LNP [ $p K a$ 7.15; Figure 6, lower]). However, we observed that traces of the YSK05/12-LNP were distributed into hepatocytes and liver resident macrophages (Kupffer cells). Furthermore, the biodistribution of YSK05/12-LNP to the lung and spleen was minimal (only a weak siRNA fluorescent intensity was detected in lungs [Figure S2]) and its silencing activity in lung and spleen endothelia was weak (Figure S1). The selectivity of the YSK05/12-LNP for the liver endothelium over the lung or spleen endothelium can be attributed to the special scavenging character of LSECs, which have a very high endocytic capacity and are responsible for the uptake of soluble or colloidal materials and nanoparticles that are not large enough to be phagocytosed by Kupffer cells. ${ }^{16,17,20,46,47}$ It is noteworthy that, despite the improved activity in LSECs, the YSK05-LNP was still stronger in hepatocytes than the YSK05/12-LNP in LSECs. This can be attributed to the difference in the type of siRNA used to target hepatocytes or LSECs (siFVII vs siCD31, respectively), which might have different gene silencing strengths. Furthermore, differences in cellular properties between hepatocytes and LSECs should also be considered. Another possible explanation is related to the low uptake of YSK05/12-LNP by Kupffer cells due to its cationic charge. However, this uptake is small compared with that of YSK12-C4-LNP ( $p K a$ 8.00), which could be taken up by Kupffer cells due to its highly cationic charge, and despite its strong activity in vitro, ${ }^{36}$ it lost its activity completely in vivo. Moreover, the weak activity of the YSK12-C4-LNP could be attributed to its rapid disintegration in the blood stream and the leakage of siRNA. As shown in Figure S2, the siRNA was rapidly eliminated and detected in the kidney at 30 minutes after intravenous administration, possibly due to the high cationic charge of the LNP and its recognition by the reticuloendothelial system, while the lipids derived from LNPs were mostly distributed to lungs and, to a lower extent, the liver. This suggests that the $p K a$ value of the LNP is critical in terms of its pharmacokinetics and pharmacodynamics and should be used in conjunction with a cation to maintain the LNP's integrity, stability in the blood, and distribution and uptake by targeted tissues.

The optimized YSK05/12-LNP ( $p K a$ 7.15) induced an mRNA knockdown of approximately $60 \%$ in the case of a $0.1 \mathrm{mg} / \mathrm{kg}$ dose of siRNA targeting the CD31 gene in LSECs. This is a remarkable improvement compared with YSK05-LNP, which induced only around 20\% knockdown in LSECs with the same dose, and to the YSK12-C4-LNP which induced no activity at all with the same dose. In addition, this LNP had a stronger activity than a previously optimized LNP in our laboratory to target LSECs, in which the YSK05-LNP was modified by attaching a ligand specific for LSECs to its surface (the KLGR peptide ligand was used which was designed from the ApoB-100 sequence of an LDL molecule), and which needed a higher dose of $1 \mathrm{mg}$ siRNA/ $\mathrm{kg}$ to induce effective gene silencing. ${ }^{48,49}$

The $p K a$ value of the LNP 7.15 was determined to be a balanced value, not low or neutral in order to be taken up by hepatocytes, and not very cationic in order to be taken up by Kupffer cells. In contrast, a $p K a$ value of 7.15 represents a $\sim 36 \%$ cationic charge, which was found to be optimum for LSECs uptake (Figure 8). The YSK05/12-LNP is free of toxicity and side effects (Figure 7), which makes it a better substitute for viral vectors. ${ }^{50}$ In addition, it has potential applications for producing in vivo pharmacological effects by silencing any gene of interest in LSECs, thus permitting a variety of diseases associated with such cells to be treated. Unlike ligandbased, ${ }^{51-54}$ or size-based LSECs targeting, ${ }^{55}$ the YSK05/12-LNP provided, for the first time, a new strategy for targeting LSECs through optimizing the $p K a$ value of the carrier membrane or ionization property, which was confirmed to be an important physiochemical property that could have great applications in targeting more challenging tissues other than the liver. Further studies are needed to investigate the physiochemical properties of the $p K a$-modified LNPs in the physiological environment and their biophysical interactions with protein corona which could alter their fate and interaction with targeted cellular membranes, as discussed previously elsewhere. ${ }^{56}$ 
YSK05-LNP

(neutral, pKa 6.50)

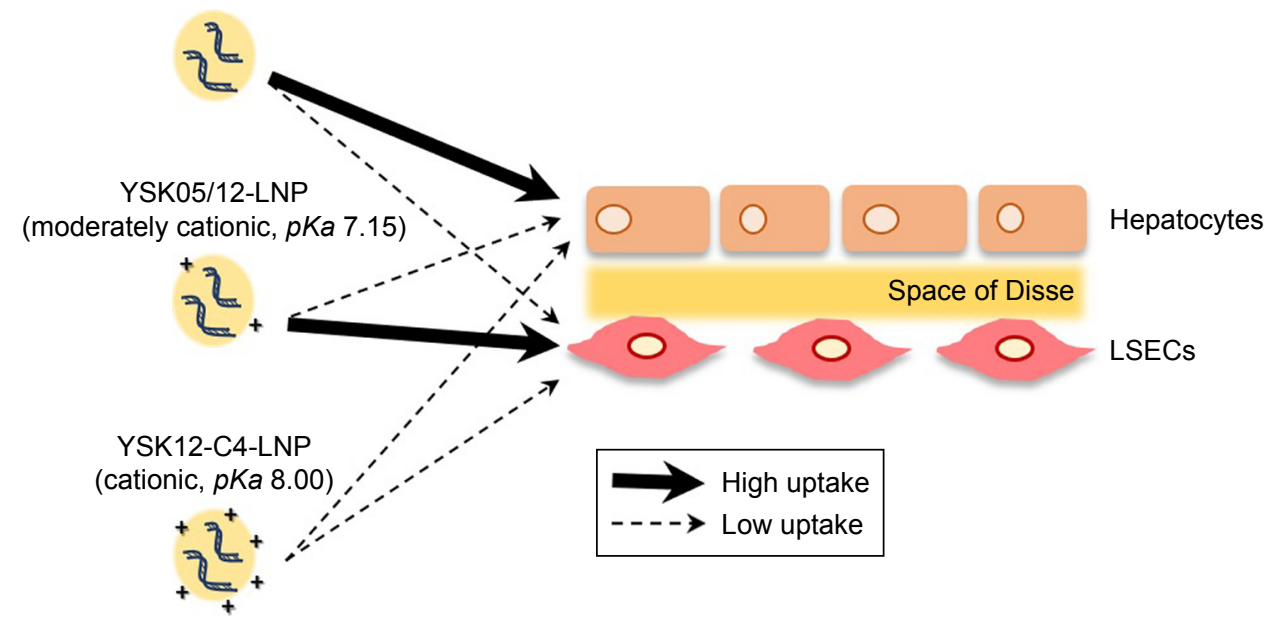

Figure 8 Schematic illustration of the current strategy and findings.

Note: Manipulating the $p K a$ value of the LNP's membrane for optimizing the targeting of LSECs.

Abbreviations: LNP, lipid nanoparticle; LSECs, liver sinusoidal endothelial cells; pKa, acid dissociation constant.

\section{Conclusion}

In the present study, specific tissue targeting was successfully achieved by optimizing the $p K a$ value of the membrane of an LNP by mixing different lipids with different $p K a$ values into one formulation. The lipids used in this study did not contribute equally to the process, in that a nonlinear pattern of $p K a$ modification was found, in which the final $p K a$ of the LNP membrane was dependent not only on the quantity of each lipid but also on the individual contribution of each lipid in the final $p K a$ value. This strategy has the potential for preparing custom LNPs with endless varieties of structures and final $p K a$ values, and would have potential applications in drug delivery and ionic-based tissue targeting.

\section{Acknowledgments}

This work was supported by a grant from the Japan Society for Promotion of Science (JSPS) KAKENHI (grant numbers $15 \mathrm{~K} 20831$ and 17H05052). The authors also wish to thank doctor Milton S Feather for his helpful advice in writing the English manuscript.

\section{Disclosure}

The authors report no conflicts of interest in this work.

\section{References}

1. Matsumura Y, Maeda $\mathrm{H}$. A new concept for macromolecular therapeutics in cancer chemotherapy: mechanism of tumoritropic accumulation of proteins and the antitumor agent smancs. Cancer Res. 1986;46(12 Pt 1): 6387-6392.

2. Maeda H, Matsumura Y. Tumoritropic and lymphotropic principles of macromolecular drugs. Crit Rev Ther Drug Carrier Syst. 1989; 6(3):193-210.
3. VitettaES, KrolickKA, Miyama-Inaba M, Cushley W, Uhr JW.Immunotoxins: a new approach to cancer therapy. Science. 1983;219(4585):644-650.

4. Byrne JD, Betancourt T, Brannon-Peppas L. Active targeting schemes for nanoparticle systems in cancer therapeutics. Adv Drug Deliv Rev. 2008;60(15):1615-1626.

5. Torchilin VP, Zhou F, Huang L. pH-sensitive liposomes. J Liposome Res. 1993;3(2):201-255.

6. Weinstein JN, Magin RL, Yatvin MB, Zaharko DS. Liposomes and local hyperthermia: selective delivery of methotrexate to heated tumors. Science. 1979;204(4389):188-191.

7. Alexis F, Pridgen E, Molnar LK, Farokhzad OC. Factors affecting the clearance and biodistribution of polymeric nanoparticles. Mol Pharm. 2008;5(4):505-515.

8. Nigavekar SS, Sung LY, Llanes M, et al. 3H dendrimer nanoparticle organ/tumor distribution. Pharm Res. 2004;21(3):476-483.

9. Kumar P, Ban HS, Kim SS, et al. T cell-specific siRNA delivery suppresses HIV-1 infection in humanized mice. Cell. 2008;134(4):577-586.

10. Eguchi A, Meade BR, Chang YC, et al. Efficient siRNA delivery into primary cells by a peptide transduction domain-dsRNA binding domain fusion protein. Nat Biotechnol. 2009;27(6):567-571.

11. Singh Y, Tomar S, Khan S, et al. Bridging small interfering RNA with giant therapeutic outcomes using nanometric liposomes. $J$ Control Release. 2015;220(Pt A):368-387.

12. Leung AK, Tam YY, Cullis PR. Lipid nanoparticles for short interfering RNA delivery. Adv Genet. 2014;88:71-110.

13. Hope MJ. Enhancing siRNA delivery by employing lipid nanoparticles. Ther Deliv. 2014;5(6):663-673.

14. Wan C, Allen TM, Cullis PR. Lipid nanoparticle delivery systems for siRNA-based therapeutics. Drug Deliv Transl Res. 2014;4(1):74-83.

15. Maier MA, Jayaraman M, Matsuda S, et al. Biodegradable lipids enabling rapidly eliminated lipid nanoparticles for systemic delivery of RNAi therapeutics. Mol Ther. 2013;21(8):1570-1578.

16. Wisse E. An electron microscopic study of the fenestrated endothelial lining of rat liver sinusoids. J Ultrastruct Res. 1970;31(1):125-150.

17. Wisse E. An ultrastructural characterization of the endothelial cell in the rat liver sinusoid under normal and various experimental conditions, as a contribution to the distinction between endothelial and Kupffer cells. J Ultrastruct Res. 1972;38(5):528-562.

18. Fraser R, Bosanquet AG, Day WA. Filtration of chylomicrons by the liver may influence cholesterol metabolism and atherosclerosis. Atherosclerosis. 1978;29(2):113-123. 
19. Redgrave TG. Formation of cholesteryl ester-rich particulate lipid during metabolism of chylomicrons. J Clin Invest. 1970;49(3):465-471.

20. Smedsrød B, Pertoft H, Gustafson S, Laurent TC. Scavenger functions of the liver endothelial cell. Biochem J. 1990;266(2):313-327.

21. Shah V, Haddad FG, Garcia-Cardena G, et al. Liver sinusoidal endothelial cells are responsible for nitric oxide modulation of resistance in the hepatic sinusoids. J Clin Invest. 1997;100(11):2923-2930.

22. Deleve LD, Wang X, Guo Y. Sinusoidal endothelial cells prevent rat stellate cell activation and promote reversion to quiescence. Hepatology 2008;48(3):920-930.

23. Schaffner F, Poper H. Capillarization of hepatic sinusoids in man. Gastroenterology. 1963;44:239-242.

24. Thabut D, Shah V. Intrahepatic angiogenesis and sinusoidal remodeling in chronic liver disease: new targets for the treatment of portal hypertension? J Hepatol. 2010;53(5):976-980.

25. Ramadori G, Moriconi F, Malik I, Dudas J. Physiology and pathophysiology of liver inflammation, damage and repair. J Physiol Pharmacol. 2008;59:107-117.

26. Neubauer K, Wilfling T, Ritzel A, Ramadori G. Platelet-endothelial cell adhesion molecule-1 gene expression in liver sinusoidal endothelial cells during liver injury and repair. J Hepatol. 2000;32(6):921-932.

27. Márquez J, Kohli M, Arteta B, et al. Identification of hepatic microvascular adhesion-related genes of human colon cancer cells using random homozygous gene perturbation. Int J Cancer. 2013;133(9):2113-2122.

28. Pasarín M, La Mura V, Gracia-Sancho J, et al. Sinusoidal endothelial dysfunction precedes inflammation and fibrosis in a model of NAFLD. PLoS One. 2012;7(4):e32785.

29. Wu Z, Han M, Chen T, Yan W, Ning Q. Acute liver failure: mechanisms of immune-mediated liver injury. Liver Int. 2010;30(6):782-794.

30. Mori T, Okanoue T, Sawa Y, Hori N, Ohta M, Kagawa K. Defenestration of the sinusoidal endothelial cell in a rat model of cirrhosis. Hepatology. 1993;17(5):891-897.

31. Arteta B, Lasuen N, Lopategi A, Sveinbjörnsson B, Smedsrød B, Vidal-Vanaclocha F. Colon carcinoma cell interaction with liver sinusoidal endothelium inhibits organ-specific antitumor immunity through interleukin-1-induced mannose receptor in mice. Hepatology. 2010;51(6):2172-2182.

32. Sato Y, Hatakeyama H, Hyodo M, Harashima H. Relationship between the physicochemical properties of lipid nanoparticles and the quality of siRNA delivery to liver cells. Mol Ther. 2016;24(4):788-795.

33. Sato Y, Hatakeyama H, Sakurai Y, Hyodo M, Akita H, Harashima H. A pH-sensitive cationic lipid facilitates the delivery of liposomal siRNA and gene silencing activity in vitro and in vivo. $J$ Control Release. 2012;163(3):267-276.

34. Yamamoto N, Sato Y, Munakata T, et al. Novel pH-sensitive multifunctional envelope-type nanodevice for siRNA-based treatments for chronic HBV infection. J Hepatol. 2016;64(3):547-555.

35. Sato Y, Hatakeyama H, Hyodo M, Akita H, Harashima H. Development of an efficient short interference RNA (siRNA) delivery system with a new pH-sensitive cationic lipid. Yakugaku Zasshi. 2012;132(12):1355-1363.

36. Warashina S, Nakamura T, Sato Y, et al. A lipid nanoparticle for the efficient delivery of siRNA to dendritic cells. J Control Release. 2016; 225:183-191.

37. Watanabe T, Hatakeyama $\mathrm{H}$, Matsuda-Yasui $\mathrm{C}$, et al. In vivo therapeutic potential of Dicer-hunting siRNAs targeting infectious hepatitis $\mathrm{C}$ virus. Sci Rep. 2014;4:4750.

38. Matsui H, Sato Y, Hatakeyama H, Akita H, Harashima H. Sizedependent specific targeting and efficient gene silencing in peritoneal macrophages using a $\mathrm{pH}$-sensitive cationic liposomal siRNA carrier Int J Pharm. 2015;495(1):171-178.

39. Bailey AL, Cullis PR. Modulation of membrane fusion by asymmetric transbilayer distributions of amino lipids. Biochemistry. 1994;33(42): 12573-12580.
40. Zhang J, Fan H, Levorse DA, Crocker LS. Ionization behavior of amino lipids for siRNA delivery: determination of ionization constants, SAR, and the impact of lipid $\mathrm{pKa}$ on cationic lipid-biomembrane interactions. Langmuir. 2011;27(5):1907-1914.

41. Yasuda T, Ishida T, Rader DJ. Update on the role of endothelial lipase in high-density lipoprotein metabolism, reverse cholesterol transport, and atherosclerosis. Circ J. 2010;74(11):2263-2270.

42. Habrant D, Peuziat P, Colombani T, et al. Design of ionizable lipids to overcome the limiting step of endosomal escape: application in the intracellular delivery of mRNA, DNA, and siRNA. J Med Chem. 2016; 59(7):3046-3062

43. Jayaraman M, Ansell SM, Mui BL, et al. Maximizing the potency of siRNA lipid nanoparticles for hepatic gene silencing in vivo. Angew Chem Int Ed Engl. 2012;51(34):8529-8533.

44. Annema W, Tietge UJ. Role of hepatic lipase and endothelial lipase in high-density lipoprotein-mediated reverse cholesterol transport. Curr Atheroscler Rep. 2011;13(3):257-265.

45. Kajimoto K, Sato Y, Nakamura T, Yamada Y, Harashima H. Multifunctional envelope-type nano device for controlled intracellular trafficking and selective targeting in vivo. J Control Release. 2014;190: 593-606.

46. Smedsrød B, Le Couteur D, Ikejima K, et al. Hepatic sinusoidal cells in health and disease: update from the 14th International Symposium. Liver Int. 2009;29(4):490-501.

47. Elvevold K, Smedsrød B, Martinez I. The liver sinusoidal endothelial cell: a cell type of controversial and confusing identity. Am J Physiol Gastrointest Liver Physiol. 2008;294(2):G391-G400.

48. Akhter A, Hayashi Y, Sakurai Y, Ohga N, Hida K, Harashima H. A liposomal delivery system that targets liver endothelial cells based on a new peptide motif present in the ApoB-100 sequence. Int J Pharm. 2013;456(1):195-201.

49. Akhter A, Hayashi Y, Sakurai Y, Ohga N, Hida K, Harashima H. Ligand density at the surface of a nanoparticle and different uptake mechanism: two important factors for successful siRNA delivery to liver endothelial cells. Int J Pharm. 2014;475(1-2):227-237.

50. Abel T, El Filali E, Waern J, et al. Specific gene delivery to liver sinusoidal and artery endothelial cells. Blood. 2013;122(12):2030-2038.

51. Takei Y, Maruyama A, Ferdous A, et al. Targeted gene delivery to sinusoidal endothelial cells: DNA nanoassociate bearing hyaluronanglycocalyx. FASEB J. 2004;18(6):699-701.

52. Kren BT, Unger GM, Sjeklocha L, et al. Nanocapsule-delivered Sleeping Beauty mediates therapeutic Factor VIII expression in liver sinusoidal endothelial cells of hemophilia A mice. J Clin Invest. 2009; 119(7):2086-2099.

53. Bartsch M, Weeke-Klimp AH, Meijer DK, Scherphof GL, Kamps JA. Massive and selective delivery of lipid-coated cationic lipoplexes of oligonucleotides targeted in vivo to hepatic endothelial cells. Pharm Res. 2002;19(5):676-680.

54. Bartsch M, Weeke-Klimp AH, Morselt HW, et al. Optimized targeting of polyethylene glycol-stabilized anti-intercellular adhesion molecule 1 oligonucleotide/lipid particles to liver sinusoidal endothelial cells. Mol Pharmacol. 2005;67(3):883-890.

55. Khan OF, Zaia EW, Yin H, et al. Ionizable amphiphilic dendrimer-based nanomaterials with alkyl-chain-substituted amines for tunable siRNA delivery to the liver endothelium in vivo. Angew Chem Int Ed Engl. 2014;53(52):14397-14401.

56. Nguyen VH, Lee BJ. Protein corona: a new approach for nanomedicine design. Int J Nanomedicine. 2017;12:3137-3151. 


\section{Supplementary materials}

Table SI List of siRNA sequences used in this study

\begin{tabular}{l|l|l}
\hline siRNA & Sense strand $\left(5^{\prime}-\mathbf{3}^{\prime}\right)$ & Antisense strand (5'-3') \\
\hline siFVII & GGAucAucucAAGucuuACT*T & GuAAGAcuuGAGAuGAuccT*T \\
siCD3I-I & GCACAGUGAUGCUGAACAATT & UUGUUCAGCAUCACUGUGCTT \\
siCD3I-2 & GUGCAUAGUUCAAGUGACATT & UGUCACUUGAACUAUGCACTT \\
siCD3I-3 & GCAAGAAGCAGGAAGGACATT & UGUCCUUCCUGCUUCUUGCTT \\
Cy5-siGFP & AcAuGAAGCAGcACGACuUT*T & AAGUCGUGCUGCUUCAUGUT*T-Cy5 \\
AF647-siGL4 & AF647-CCGUCGUCUUCGUGAACAATT & UUGCUCACGAAUACGACGGTT \\
siGL4 & CCGUCGUCUUCGUGAACAATT & UUGCUCACGAAUACGACGGTT \\
\hline
\end{tabular}

Notes: 2 '-OMe-modified nucleotides are depicted in lowercase letters, 2 '-fluoro-modified nucleotides are shown in bold lowercase letters, and phosphorothioate linkages are denoted by asterisks. The siCD3I-I, -2, and -3 were mixed together in advance.

Abbreviations: AF647, alexafluor 647; FVII, coagulation factor VII; GFP, green fluorescent protein; siRNA, short interfering RNA.

Table S2 List of PCR primers used in this study

\begin{tabular}{l|l|l}
\hline Gene & Forward $\left(\mathbf{5}^{\prime} \mathbf{\prime} \mathbf{3}^{\prime} \mathbf{)}\right.$ & Reverse $\left(\mathbf{5}^{\prime} \mathbf{- \mathbf { 3 } ^ { \prime } \mathbf { ) }}\right.$ \\
\hline GAPDH & AGCAAGGACACTGAGCAAG & TAGGCCCCTCCTGTTATTATG \\
ACTB & AGAGGGAAATCGTGCGTGAC & CAATAGTGATGACCTGGCCGT \\
CD3I & TACAGTGGACACTACACCTG & GACTGGAGGAGAACTCTAAC \\
TIE2 & GACCAGACTGTAAGCTCAG & AATCCTCTATCTGTGGAGTC \\
\hline
\end{tabular}

Abbreviations: ACTB, beta-actin; GAPDH, glyceraldehyde 3-phosphate dehydrogenase; PCR, polymerase chain reaction; TIE2, angiopoietin receptor.

Table S3 Physical characteristics of the LNPs

\begin{tabular}{|c|c|c|c|c|c|c|c|}
\hline \multicolumn{2}{|c|}{ Lipid composition } & \multirow[t]{2}{*}{$p K a$} & \multirow[t]{2}{*}{ Size (nm) } & \multirow{2}{*}{$\begin{array}{l}\text { Number } \\
\text { mean }(\mathrm{nm})\end{array}$} & \multirow[t]{2}{*}{ PDI } & \multirow{2}{*}{$\begin{array}{l}\zeta \text {-potential } \\
(\mathrm{mV})\end{array}$} & \multirow{2}{*}{$\begin{array}{l}\text { EE of } \\
\text { siRNA (\%) }\end{array}$} \\
\hline $\begin{array}{l}\text { YSK05 } \\
\text { (mol\%) } \\
\end{array}$ & \begin{tabular}{|l} 
YSK I 2-C4 \\
(mol\%)
\end{tabular} & & & & & & \\
\hline 70 & 0 & 6.50 & $84.02 \pm 6.82$ & $58.52 \pm 3.49$ & $0.13 \pm 0.03$ & $1.70 \pm 4.59$ & $94.82 \pm 0.64$ \\
\hline 50 & 20 & 7.15 & $85.59 \pm 8.22$ & $55.71 \pm 2.91$ & $0.17 \pm 0.08$ & $6.12 \pm 1.46$ & $95.32 \pm 1.12$ \\
\hline 35 & 35 & 7.50 & $84.89 \pm 11.79$ & $56.31 \pm 7.80$ & $0.14 \pm 0.08$ & $7.75 \pm 2.83$ & $96.18 \pm 1.73$ \\
\hline 20 & 50 & 7.75 & $92.86 \pm 10.27$ & $56.55 \pm 8.01$ & $0.19 \pm 0.04$ & $8.97 \pm 1.74$ & $97.58 \pm 0.96$ \\
\hline 0 & 70 & 8.00 & $92.61 \pm 11.47$ & $51.72 \pm 0.99$ & $0.22 \pm 0.10$ & $11.63 \pm 1.56$ & $97.41 \pm 0.67$ \\
\hline
\end{tabular}

Notes: After preparing LNPs that have the composition YSK05/YSK I2 C4/Cholesterol/ ${ }_{\mathrm{m}} \mathrm{PEG}_{2 \mathrm{k}}{ }_{\mathrm{k}} \mathrm{DMG}=(70-X): X: 30: 2 \mathrm{~mol} \%$ of total lipids $(X=0 \sim 70)$ by $t$-BuOH dilution method, the pKa was measured using a TNS assay; size, number mean, PDI, and $\zeta$-potential were measured using DLS; EE of siRNA was measured using RiboGreen assay. Data represent mean $\pm S D(n=3)$.

Abbreviations: DLS, dynamic light scattering; EE, encapsulation efficiency; LNP, lipid nanoparticle; PDI, polydispersity index; ${ }_{m}$ PEG $_{2 k}$-DMG, I,2-Dimirystoyl-sn-3-glycero methoxypolyethyleneglycol 2000 ether; $p K a$, acid dissociation constant; siRNA, short interfering RNA; $t$-BuOH, tertiary butanol; TNS, 6-(p-toluidino)-2-naphthalenesulfonic acid.
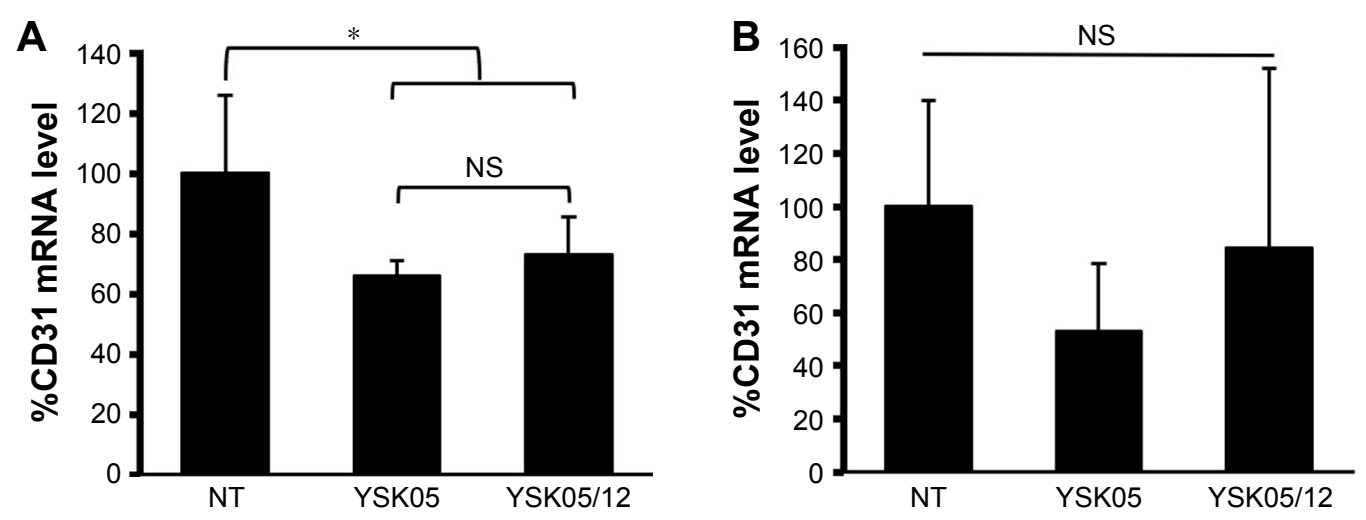

Figure SI In vivo silencing activity of YSK05-LNP (pKa 6.50) and YSK05/I2-LNP (pKa 7.I5) in the lung and spleen.

Notes: Mice were intravenously injected with the LNPs encapsulating a mixture of siRNA (siCD3I:siFVII I:I ratio) at a dose of 0.1 mg/kg of each type of siRNA. After 24 hours, the level of CD3I mRNA in the lung $(\mathbf{A})$ and spleen $(\mathbf{B})$ was measured by means of qRT-PCR. The reference genes were TIE2 in the lung and GAPDH in the spleen. $* P<0.05$, nonrepeated ANOVA followed by an SNK test; data represent mean \pm SD $(n=4)$.

Abbreviations: FVII, coagulation factor VII; GAPDH, glyceraldehyde 3-phosphate dehydrogenase; LNP, lipid nanoparticle; mRNA, messenger RNA; NS, not significant; NT, non-treated; $p K a$, acid dissociation constant; qRT-PCR, quantitative real-time polymerase chain reaction; TIE2, angiopoietin receptor. 

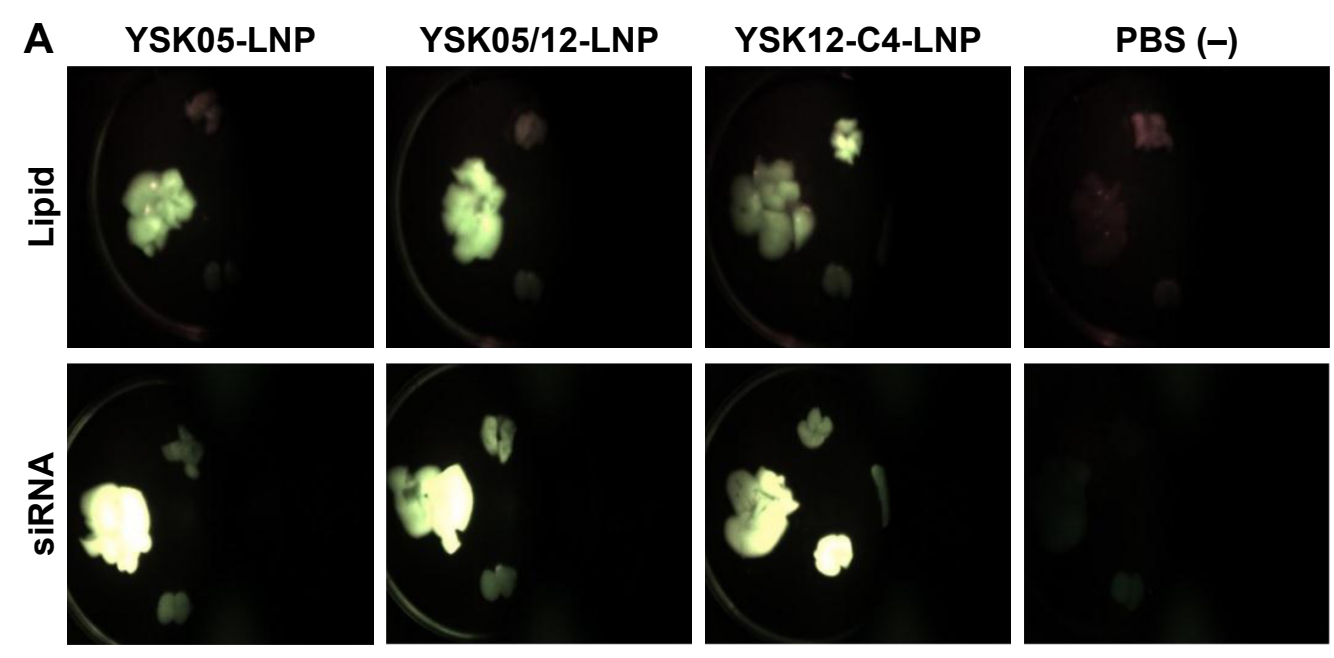

Organ position
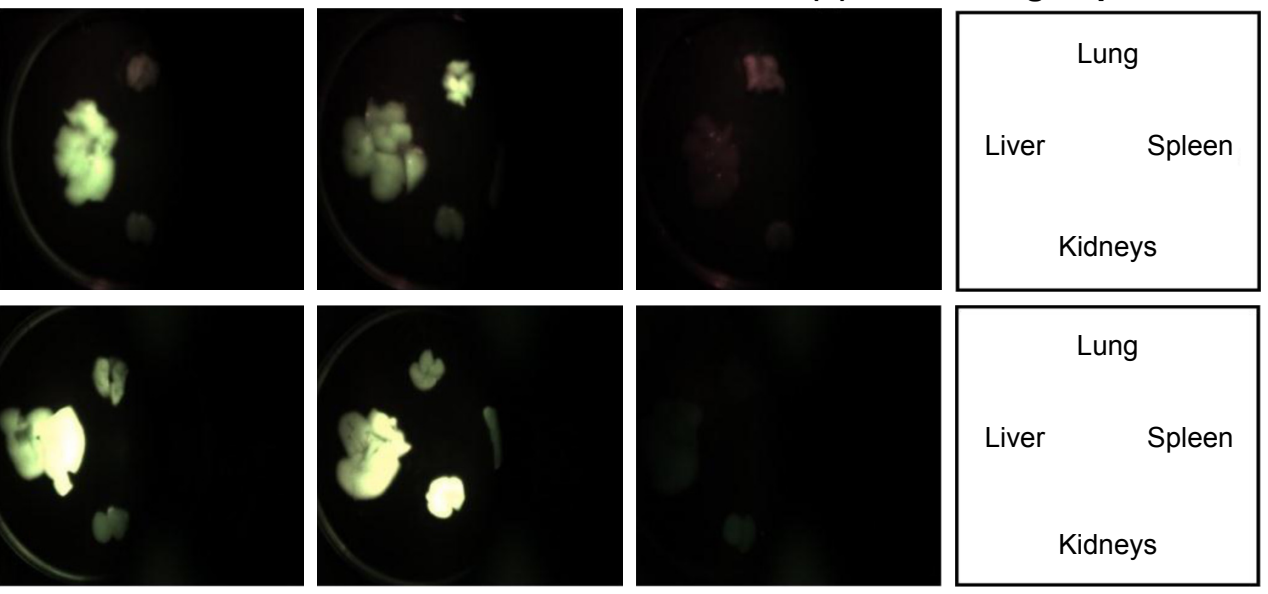

B

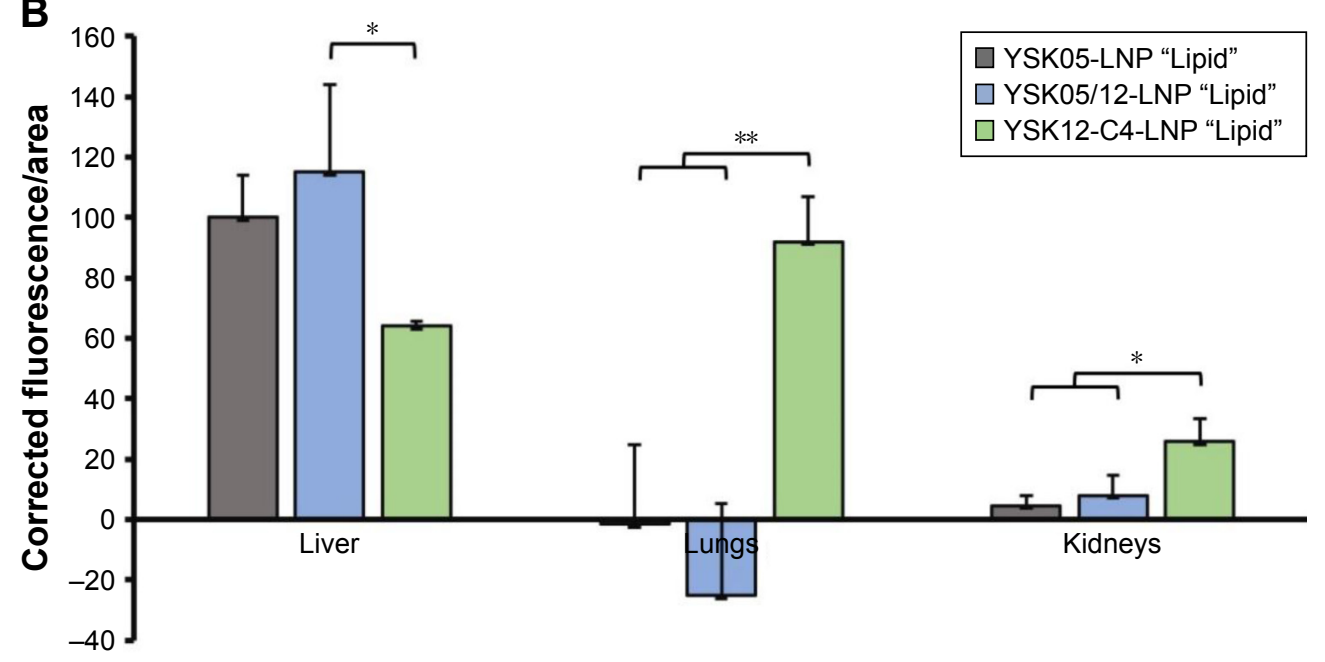

C

$$
\text { C }
$$

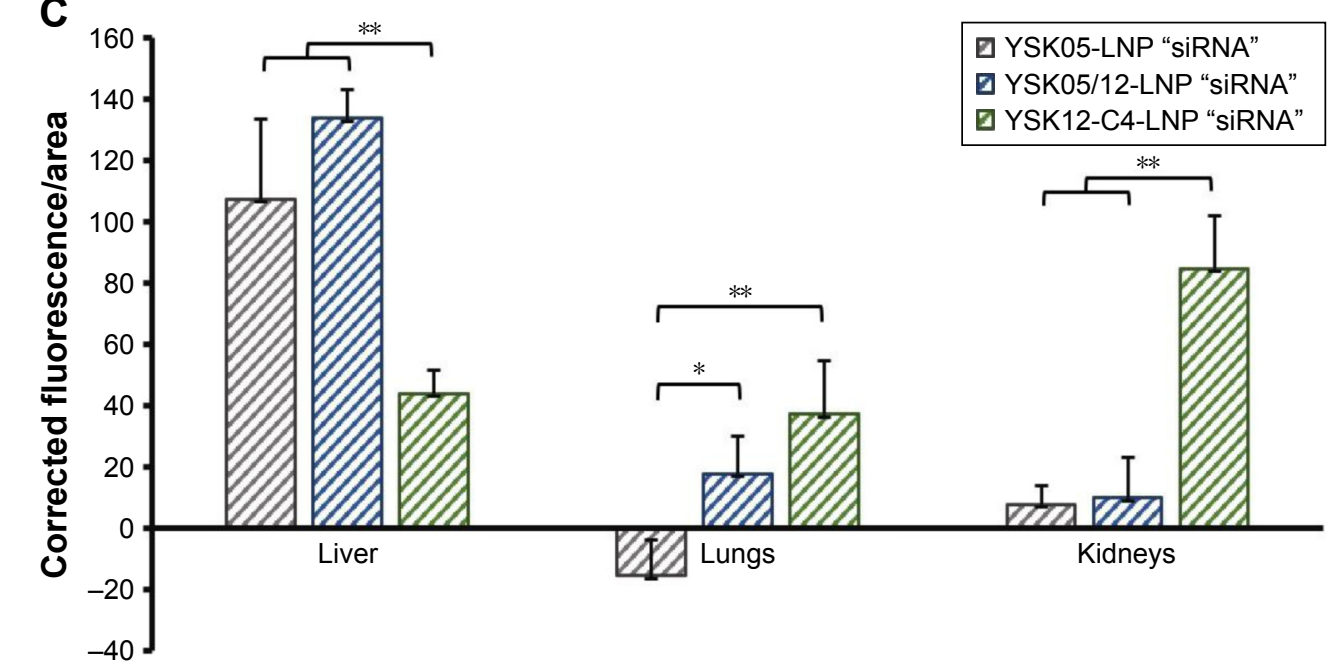

๑ YSK05-LNP "SiRNA"

口 YSK05/12-LNP "SiRNA"

$\square$ YSK12-C4-LNP "siRNA"

Figure S2 Biodistribution of LNPs.

Notes: Mice were intravenously injected, regardless of their body weight, with $200 \mu \mathrm{L}$ of LNPs encapsulating $10 \mu \mathrm{g}$ siRNA (assuming a mouse body weight of 20 g, the injected dose will be $0.5 \mathrm{mg}$ siRNA/kg). In the LNPs, either the lipids were labeled with DiD or the siRNA was labeled with AF647. At 30 minutes after injection, the mice were sacrificed and body organs (liver, lung, kidneys, and spleen) were collected and fluorescence of either the lipids or the siRNA was observed using FluorVivo 300 small animal fluorescence imaging (A). The biodistribution of lipids (B) and siRNA (C), respectively, was further quantified using Image software. $* P<0.05$, $* * P<0.0$ I, nonrepeated ANOVA followed by an SNK test; data represent the mean \pm SD $(n=3)$.

Abbreviations: AF647, alexafluor 647; LNP, lipid nanoparticle; siRNA, short interfering RNA. 


\section{Publish your work in this journal}

The International Journal of Nanomedicine is an international, peerreviewed journal focusing on the application of nanotechnology in diagnostics, therapeutics, and drug delivery systems throughout the biomedical field. This journal is indexed on PubMed Central, MedLine, CAS, SciSearch $\AA$, Current Contents $\AA /$ Clinical Medicine,

Journal Citation Reports/Science Edition, EMBase, Scopus and the Elsevier Bibliographic databases. The manuscript management system is completely online and includes a very quick and fair peer-review system, which is all easy to use. Visit http://www.dovepress.com/ testimonials.php to read real quotes from published authors.

Submit your manuscript here: http://www.dovepress.com/international-journal-of-nanomedicine-journal 